\title{
Fixed points, Koebe uniformization and circle packings
}

\author{
By Zheng-Xu He and Oded Schramm*
}

\section{Contents}

Introduction

1. The space of boundary components

2. The fixed-point index

3. The Uniqueness Theorem

4. The Schwarz-Pick lemma

5. Extension to the boundary

6. Maximum modulus, normality and angles

7. Uniformization

8. Domains in Riemann surfaces

9. Uniformizations of circle packings Addendum

\section{Introduction}

A domain in the Riemann sphere $\hat{\mathbb{C}}$ is called a circle domain if every connected component of its boundary is either a circle or a point. In 1908, P. Koebe [Ko1] posed the following conjecture, known as Koebe's Kreisnormierungsproblem: Any plane domain is conformally homeomorphic to a circle domain in $\hat{\mathbb{C}}$. When the domain is simply connected, this is the content of the Riemann mapping theorem. The conjecture was proved for finitely connected domains and certain symmetric domains by Koebe himself ([Ko2], [Ko3]); for domains with various conditions on the "limit boundary components" by R. Denneberg [De], H. Grötzsch [Gr], L. Sario [Sa], H. Meschowski

*The authors were supported by N.S.F. Grants DMS-9006954 and DMS-9112150, respectively. The authors express their thanks to Mike Freedman, Dennis Hejhal, Al Marden, Curt McMullen, Burt Rodin, Steffen Rohde and Bill Thurston for conversations relating to this work. Also thanks are due to the referee, and to Steffen Rohde, for their careful reading and subsequent corrections.

The paper of Sibner [Si3] served as a very useful introduction to the subject. 
([Me1], [Me2]), K.L. Strebel ([Str1], [Str2]), L. Bers [Be], A. Haas [Haa] and others; and for domains quasiconformally homeomorphic to a circle domain by R.J. Sibner [Si1], [Si2].

In this article we prove the following theorem:

TheOREM 0.1. Any domain $\Omega$ in $\hat{\mathbb{C}}$, whose boundary $\partial \Omega$ has at most countably many components, is conformally homeomorphic to a circle domain $\Omega^{*}$ in $\hat{\mathbb{C}}$. Moreover $\Omega^{*}$ is unique up to Möbius transformations, and every conformal automorphism of $\Omega^{*}$ is the restriction of a Möbius transformation.

The uniqueness of $\Omega^{*}$ in the above theorem can fail if $\Omega$ has uncountably many boundary components. The theory of quasiconformal maps and the Beltrami equation (cf. [LV]) can be used to show that the complement of a Cantor set in $\hat{\mathbb{C}}$ of nonzero area provides such an example. This is done by placing a nonzero Beltrami differential supported on the Cantor set and solving the Beltrami equation to obtain a quasiconformal map which is conformal outside the Cantor set.

A circle domain in a Riemann surface is a domain, whose complement's connected components are all closed geometric disks and points. Here a geometric disk (or, in short, a disk) means a topological disk, whose lifts in the universal cover of the Riemann surface (which is the hyperbolic plane, the euclidean plane or the sphere) are round. As a consequence of Theorem 0.1 we have the following theorem:

THEOREM 0.2. Let $\Omega$ be an open Riemann surface with finite genus and at most countably many ends. Then there is a closed Riemann surface $R^{*}$ such that $\Omega$ is conformally homeomorphic to a circle domain $\Omega^{*}$ in $R^{*}$. Moreover the pair $\left(R^{*}, \Omega^{*}\right)$ is unique up to conformal homeomorphisms.

Circle domains are closely related to circle packings, and results similar to the above hold for circle packings. Recall that a circle packing $P$ is a collection of closed geometric disks with disjoint interiors. The (tangency) graph, or nerve, of a circle packing $P$ is a graph, whose vertices are in one-to-one correspondence with the packed sets, and an edge appears in the graph if and only if the corresponding disks are tangent. The carrier of a circle packing $P$ is the union of the packed disks and the finite interstices (the connected components of the complement, whose boundaries lie on finitely many of the packed disks). The circle packing theorem says that, for any triangulation $\mathcal{T}$ of the 2-sphere, there is a circle packing in $\hat{\mathbb{C}}$, unique up to Möbius transformations, whose graph is combinatorially equivalent to the 1-skeleton of $\mathcal{T}$. This theorem was first discovered by Koebe [Ko4], who obtained it as a limiting case for his uniformization theorem of finitely connected domains as circle 
domains. ${ }^{1}$ But the circle packing theorem was unnoticed, or forgotten, until W.P. Thurston [Th1] rediscovered it as a corollary of E.M. Andreev's theorem ([An1], [An2]). Thurston [Th2] then conjectured that finite circle packings can approximate the Riemann map from a simply connected domain to the unit disk. This conjecture was proved by B. Rodin and D. Sullivan [RoSu], and much research on circle packings followed.

A slightly modified proof of Theorem 0.1 yields the following generalization of the circle packing theorem to infinite triangulations:

THEOREM 0.3. Let $\mathcal{T}$ be a triangulation of a domain in $\hat{\mathbb{C}}$ with at most countably many boundary components. Then there is a circle packing $P$ in $\hat{\mathbb{C}}$ whose graph is combinatorially equivalent to the 1-skeleton of $\mathcal{T}$ and whose carrier is a circle domain. Moreover $P$ is unique up to Möbius transformations.

This theorem was conjectured in [Sch2]. More generally we have the following theorem:

THEOREM 0.4. Let $\mathcal{T}$ be a triangulation of a finite-genus open surface with at most countably many ends. Then there are a closed Riemann surface $R$ and a circle packing $P \subset R$ whose graph is combinatorially equivalent to the 1 -skeleton of $\mathcal{T}$ and whose carrier is a circle domain in $R$. Moreover $R$ and $P$ are unique up to conformal or anticonformal homeomorphisms.

As a special case of Theorem 0.3 we have the following corollary:

Corollary 0.5. Let $\mathcal{T}$ be a triangulation of a simply connected plane domain. Then there is a circle packing $P$ in $\mathbb{C}$ whose graph is combinatorially equivalent to the 1-skeleton of $\mathcal{T}$ and whose carrier is either the (euclidean) plane $\mathbb{C}$ or the unit disk $U$. Moreover $P$ is unique up to Möbius transformations.

The 1-skeleton of $\mathcal{T}$ is called a parabolic graph if the carrier of the circle packing $P$ of the above corollary is equal to $\mathbb{C}$; otherwise it is called a hyperbolic graph. In [HeSch] and [BSte2] some combinatorial criteria are given for determining if a graph is parabolic or hyperbolic.

The uniqueness (or rigidity) statement of Corollary 0.5 was previously proved by the second author in [Sch2]. In the restricted case, when there is a uniform upper bound on the valences of all the vertices in the triangulation $\mathcal{T}$, the existence statement of Corollary 0.5 follows from [BSte1], and the uniqueness can be obtained by the methods of [RoSu] or [He2] (see also [Ro1]).

\footnotetext{
${ }^{1}$ The authors thank Horst Sachs for this reference.
} 
This work originated with the proof of Theorem 0.3 , and then we noticed that the techniques apply to circle domains as well. However the proof of Theorem 0.1 does not mention circle packings.

Theorem 0.1 has two parts: uniqueness and existence. The uniqueness is derived from an analysis of fixed-point indices of mappings. Given a domain $\Omega \subset \mathbb{C}$ and a mapping $f: \partial \Omega \rightarrow \mathbb{C}$, the fixed-point index of $f$ can be defined as the total number of fixed points of a continuous map $F: \Omega \rightarrow \mathbb{C}$ whose restriction to $\partial \Omega$ is $f$, counting multiplicities. (One should restrict $f$ to having no fixed points, and $F$ to having only isolated fixed points.) This number does not depend on the choice of $F$. Conformal maps have only positive-multiplicity fixed points, and so, if the index of $f$ is negative, then one can conclude that there is no continuous $F$ whose restriction to $\partial \Omega$ is $f$ and whose restriction to $\Omega$ is conformal. The first fundamental observation in the proof of uniqueness is that the index of any orientation-preserving homeomorphism that takes a circle to a circle is nonnegative; thus much information is available for domains that have circles as boundary components. As was known already to Strebel [Str1], this observation implies that a conformal homeomorphism between circle domains with countably many boundary components that extends continuously to the boundary is a Möbius transformation. However Strebel was not able to prove the continuous extension to the boundary, as is done below. $^{2}$

One of the main tools we use both in the uniqueness and existence parts of Theorem 0.1 is the following Schwarz-Pick lemma for multiply connected domains:

ThEOREM 0.6 (Schwarz-Pick lemma for multiply connected domains). Let $U \subset \mathbb{C}$ denote the open unit disk and let $A$ and $A^{*}$ be Jordan domains in $\mathbb{C}$ with $A \supset U \supset A^{*}$. Let $\Omega$ be a domain, which is obtained from $A$ by the deletion of a closed disjoint union of at most countably many closed (geometric) disks and points in $A$. Similarly let $\Omega^{*}$ be a domain obtained from $A^{*}$ by the deletion of a closed disjoint union of at most countably many closed disks and points. Suppose that $f: \Omega \rightarrow \Omega^{*}$ is a conformal homeomorphism between $\Omega$ and $\Omega^{*}$ and that $f^{B}(\partial A)=\partial A^{*}$. Then $f$ is a contraction in the hyperbolic metric in the following sense: If $p, q \in \Omega \cap U$ are distinct, then

$$
d_{\text {hyp }}(f(p), f(q)) \leqslant d_{\text {hyp }}(p, q),
$$

where $d_{\text {hyp }}(\cdot, \cdot)$ denotes the distance in the hyperbolic metric of $U$. Furthermore, if equality holds for one pair $p \neq q$, then $\Omega \subset U$ and $f$ is a restriction to $\Omega$ of a hyperbolic isometry.

\footnotetext{
${ }^{2}$ The authors thank Dennis Hejhal for pointing out this work of Strebel.
} 
This is already an interesting result in the finite-connectivity case; that is, when the collection of deleted disks and points is finite. Our proof of this theorem is also based on fixed-point arguments. A version of the Schwarz-Pick lemma for circle packings was previously obtained by Rodin ([Ro1], [Ro2]) and by A. Beardon and K. Stephenson ([BSte1], [BSte3]).

Both the proof of the continuous extension to the boundary and the proof of existence use transfinite induction, where the induction is with respect to the "complexity" of the boundary, as in Sibner's paper [Si3]. We prove existence by taking limits of maps from simpler subdomains of $\Omega$. A maximum modulus principle and normality results, as well as the Schwarz-Pick lemma, are needed to conclude that the limit has the required properties.

\section{The space of boundary components}

In this section we recall the definition and properties of the space of boundary components of an open planar set.

We will use the term domain for a connected open set in the Riemann sphere $\hat{\mathbb{C}}$. Let $\Omega$ be a domain in $\hat{\mathbb{C}}$. The collection $B(\Omega)$ of boundary components of $\Omega$ has the structure of a compact Hausdorff space. One way to describe the topology on $B(\Omega)$ is the following: On the boundary of $\Omega, \partial \Omega$, consider the equivalence relation $\sim$ in which $z \sim w$ if and only if $z$ and $w$ belong to the same connected component of $\partial \Omega$. Then $B(\Omega)$ is the set of equivalence classes of $\sim, B(\Omega)=\partial \Omega / \sim$, with the quotient topology.

An intrinsic way to describe $B(\Omega)$ is as the space of ends of $\Omega$, denoted by $\mathcal{E}(\Omega)$. An end $e \in \mathcal{E}(\Omega)$ is a function that assigns to each compact subset $F \subset \Omega$ a connected component $e(F)$ of $\Omega-F$ in such a way that $e(F) \supset e\left(F^{*}\right)$ whenever $F \subset F^{*}$. The topology on the collection of ends is then defined as the minimal topology containing all sets of the form $\{e \in \mathcal{E}(\Omega): e(F)=C\}$, where $F$ is some compact subset of $\Omega$ and $C$ is some connected component of $\Omega-F$. It is then not hard to check that $B(\Omega)$ is naturally homeomorphic to the space of ends. (The end $e_{K}$ corresponding to a boundary component $K \in B(\Omega)$ is the end for which $e_{K}(F)$ is the connected component of $\Omega-F$ whose closure intersects $K$, whenever $F \subset \Omega$ is compact.) Since the space of ends is clearly independent of the embedding of $\Omega$ in $\hat{\mathbb{C}}$, we have the following well-known fact:

FACT 1.1. Let $f: \Omega \rightarrow \Omega^{*}$ be a homeomorphism of connected open sets in $\hat{\mathbb{C}}$. Then $f$ induces a canonical homeomorphism $f^{B}: B(\Omega) \rightarrow B\left(\Omega^{*}\right)$.

In the following, we shall use the notation $f^{B}$ for that homeomorphism induced by $f$. 
This article deals with domains $\Omega$, where $B(\Omega)$ is at most countable (and nonempty), and henceforth we make this assumption on $B(\Omega)$. From Baire category considerations it follows that any countable compact Hausdorff space has isolated points. Given a topological space $\mathcal{X}$, let $\mathcal{X}^{\prime}$ be $\mathcal{X}-\{$ its isolated points $\}$. Then $\mathcal{X}^{\prime}$ is closed in $\mathcal{X}$. Now, for each ordinal $\alpha$, we define $\mathcal{X}^{\alpha}$ by transfinite induction (see [Hau] for background on ordinals and transfinite induction): let $\mathcal{X}^{0}=\mathcal{X}$; for successor ordinals $\alpha=\beta+1$ let $\mathcal{X}^{\alpha}=\left(\mathcal{X}^{\beta}\right)^{\prime}$; and for limit ordinals $\alpha$ define $\mathcal{X}^{\alpha}=\bigcap_{\beta<\alpha} \mathcal{X}^{\beta}$. Then, for each ordinal $\alpha, \mathcal{X}^{\alpha}$ is closed in $\mathcal{X}$.

Since $\mathcal{X}^{\prime} \subset \mathcal{X}$, we have $\mathcal{X}^{\prime} \neq \mathcal{X}$ for every closed nonempty subset $\mathcal{X}$ of $B(\Omega)$. There is some ordinal $\beta$ so that $B(\Omega)^{\beta}=\emptyset$. Let $\gamma$ be the minimal ordinal with that property. By compactness, $\gamma$ must be a successor ordinal and $\gamma$ is, of course, countable. Let $\alpha$ be its predecessor. We will refer to $\alpha$ as the rank of $\Omega$. The rank is also defined as the only ordinal $\alpha$ such that $B(\Omega)^{\alpha}$ is finite and nonempty. If $B(\Omega)^{\alpha}$ contains $n$ points, $0<n<\infty$, then the pair $(\alpha, n)$ will be called the type of $\Omega$. For every ordinal $\beta$, an isolated point of $B(\Omega)^{\beta}$ will be called a boundary component of rank $\beta$.

\section{The fixed-point index}

Definition. Let $\gamma$ be an oriented Jordan curve in the plane $\mathbb{C}$. Let $f$ : $\gamma \rightarrow \mathbb{C}$ be a continuous map without fixed points. The (fixed-point) index of $f$, denoted by index $(f)$, is defined to be the winding number with respect to the origin 0 of the closed curve $f(z)-z$ as $z$ varies in $\gamma$. In other words, index $(f)$ is the winding number of $g \circ \gamma$ around 0 , where $g(z)=f(z)-z$ and $\gamma$ is parametrized in accordance with its orientation.

If the domain of definition of $f$ is a finite, disjoint union of oriented Jordan curves in $\mathbb{C}$ (and $f$ has no fixed points), then the index of $f$ is defined as the sum of the indices of the restrictions of $f$ to the individual curves.

Definition. Let $f: A \rightarrow \mathbb{C}$ be continuous, where $A \subset \mathbb{C}$, and suppose that $z \in \operatorname{int}(A)$ (the interior of $A$ ) is an isolated fixed point of $f$. The index of $f$ at $z$, denoted by index $(f, z)$, is defined as the index of the restriction of $f$ to $\partial D$, where $D \subset A$ is a closed disk that contains $z$ in its interior, but does not contain any other fixed point of $f$, and where $\partial D$ is positively oriented with respect to $D$. Considering homotopies makes it clear that index $(f, z)$ does not depend on the particular choice of $D$. The index of $f$ at $z$ will also be called the multiplicity of the fixed point $z$.

Theorem 2.1 (Poincaré-Hopf). Let $A \subset \mathbb{C}$ be a compact set that is the closure of its interior and whose boundary consists of finitely many disjoint 
Jordan curves. Let the boundary components of $A$ be positively oriented with respect to $A$. Suppose that $f: A \rightarrow \mathbb{C}$ is continuous, has only isolated fixed points and has no fixed points on the boundary of $A$. Then the index of the restriction of $f$ to $\partial A$ is equal to the sum of the indices of $f$ at all its fixed points.

Remarks. A simple and elementary proof of Theorem 2.1 is given below. However, for the sake of the reader whose topology is a little rusty, we will now try to put Theorem 2.1 in perspective. The common formulation of the Poincaré-Hopf theorem (cf. [Mi], [GPo]) is that on a compact smooth manifold without boundary the number of zeroes, counting multiplicities, of any continuous vector field with only isolated zeroes is equal to the Euler characteristic of the manifold. This relates to our setting in the following way: A function $f: A \rightarrow \mathbb{C}$ gives a vector field $v(z)=f(z)-z$, and the fixed points of the function $f$ correspond to the zeroes of the vector field $v$.

The boundary enters the picture in the usual manner: given a compact smooth manifold $M$ with boundary $\partial M$ and a zeroless vector field $v$ on $\partial M$ (with values taken in the tangent space of $M$, denoted by $T M$ ), the number of zeroes of a continuous extension of $v$ to all of $M$ with only isolated zeroes is independent of the extension and is therefore an invariant of the pair $(M, v)$. That is an easy consequence of the Poincaré-Hopf theorem, as follows: Let $v_{1}, v_{2}$ be two such extensions and let $M^{\prime}$ be a manifold identical to $M$, but with the reversed orientation. Glue $M$ and $M^{\prime}$ along the boundary (via the identification of $\partial M$ and $\partial M^{\prime}$ ) to form a manifold $M^{*}$ without boundary. Now $v$ is a vector field on $\partial M$ with values in $T M^{*}$. Extend it to some continuous vector field $v^{\prime}$ on $M^{\prime} \subset M^{*}$ with only isolated zeroes. Now consider two vector fields on $M^{*}: w_{1}$, which is $v_{1}$ on $M$ and $v^{\prime}$ on $M^{\prime}$, and $w_{2}$, which is $v_{2}$ on $M$ and $v^{\prime}$ on $M^{\prime}$. The Poincaré-Hopf theorem implies that the number of zeroes of $w_{1}$ and $w_{2}$ is the same, and that says that the number of zeroes of $v_{1}$ and $v_{2}$ is the same.

The Lefschetz fixed-point theorem is also very much related. Its relevant consequence here is that an orientation-preserving homeomorphism of the sphere $\hat{\mathbb{C}}$ with only isolated fixed points has, counting multiplicities, 2 fixed points. (The number 2 appears as the Euler characteristic of the sphere.) This fact is in the background of this article. For example, consider a conformal homeomorphism of $\hat{\mathbb{C}}$. By composing with a Möbius transformation, one can arrange to get at least 3 fixed points and, as we shall see below, it is impossible in this case to get fixed points with negative index. Therefore, by the Lefschetz theorem, there must be nonisolated fixed points. This says that the composition must be the identity map, and the original conformal homeomorphism must be a Möbius transformation. This simple proof that 
any conformal homeomorphism of $\hat{\mathbb{C}}$ is a Möbius transformation can be seen as the prototype for the proofs of our rigidity results below.

Proof of Theorem 2.1. Suppose first that $f$ has no fixed points. Define $F: A \rightarrow \mathbb{C}-\{0\}$ by $F(z)=f(z)-z$. The boundary of $A$, with the orientation induced by $A$, is obviously trivial in the first homology of $A$. Therefore its image under $F$ in the first homology of $\mathbb{C}-\{0\}$ is also 0 . This means that the index of the restriction of $f$ to the boundary of $A$ is 0 . And so the theorem holds in this case.

Note that the assumptions imply that $f$ will have at most finitely many fixed points. If $f$ has fixed points $z_{1}, \ldots, z_{k}$, then let $D_{1}, \ldots, D_{k}$ be small disjoint closed disks in $\operatorname{int}(A)$ containing $z_{1}, \ldots, z_{k}$ in their respective interiors. Let $A^{*}$ be the closure of $A-\bigcup_{j=1}^{k} D_{j}$. In $A^{*}$ the map $f$ has no fixed points, and so the index of the restriction of $f$ to $\partial A^{*}$ is 0 . Each $\partial D_{j}$ has the opposite orientations as a boundary component of $A^{*}$. Therefore the index of the restriction of $f$ to $\partial D_{j}$ is negated if one takes the orientation induced by $A^{*}$. We conclude that the index of the restriction of $f$ to $\partial A$ is equal to the index of the restriction of $f$ to $\bigcup_{j=1}^{k} \partial D_{j}$. The theorem follows.

A variation of the following lemma appears in [Str1].

Circle Index Lemma 2.2. Let $J, K$ be Jordan curves in $\mathbb{C}$, positively oriented with respect to the Jordan domains that they bound (in $\mathbb{C})$; and let $f: J \rightarrow K$ be an orientation-preserving homeomorphism with no fixed points. Then

(1) $\operatorname{index}(f)=\operatorname{index}\left(f^{-1}\right)$.

(2) If $J$ is contained in the closure of the Jordan domain determined by $K$, or $K$ is contained in the closure of the Jordan domain determined by $J$, then $\operatorname{index}(f)=1$.

(3) If the intersection of $K$ and $J$ contains at most 2 points, then index $(f) \geqslant 0$.

(4) If $J$ and $K$ are circles, then $\operatorname{index}(f) \geqslant 0$.

Proof. Let $g: K \rightarrow \mathbb{C}$ be defined by $g(z)=f^{-1}(z)-z$. Then the winding number around the origin of $g \circ f: J \rightarrow \mathbb{C}$ is clearly the same as that of $g$. But $g \circ f(z)=z-f(z)$, which has the same winding number around 0 as $z \rightarrow f(z)-z$. Therefore part (1) holds.

Let $\hat{J}$ and $\hat{K}$ denote the closures of the Jordan domains determined by $J$ and $K$, respectively. To prove part (2) suppose that $J \subset \hat{K}$. Let $h: J \times[0,1] \rightarrow$ $\hat{K}$ be a homotopy from the identity map of $J$ to some constant c $\in \operatorname{int}(\hat{K})$ with the property that $h(z, t) \notin K$ for $t>0$. Define $H(z, t)=f(z)-h(z, t)$ for $z \in J, t \in[0,1]$. Then 0 is not in the image of $H$, and $H$ is a homotopy from $z \rightarrow f(z)-z$ to $z \rightarrow f(z)-\mathrm{c}$. Since $\mathrm{c} \in \operatorname{int}(\hat{K})$, the map $z \rightarrow f(z)-\mathrm{c}$ 


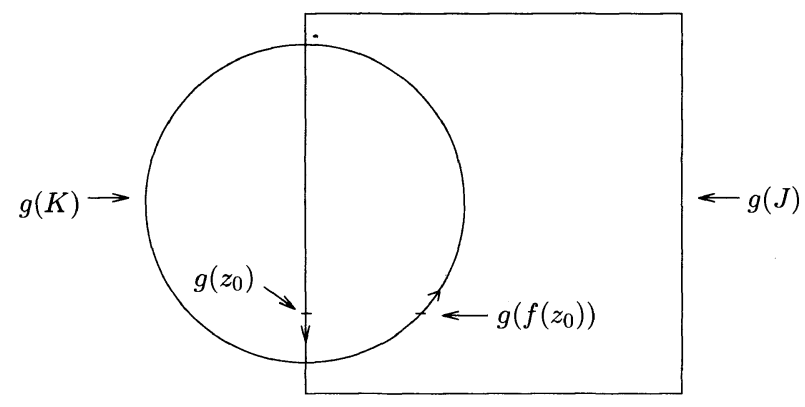

Figure 2.1. After the isotopy.

has the winding number 1 around 0 . Therefore the map $z \rightarrow f(z)-z$ has the winding number 1 around 0 , and index $(f)=1$. Using (1), we see that (2) holds.

Consider now the case where the interiors of $\hat{K}$ and $\hat{J}$ are disjoint. Let $h: J \times[0,1] \rightarrow \mathbb{C}-\operatorname{int}(\hat{K})$ be a homotopy from the identity map on $J$ to some constant $\mathrm{c} \notin \hat{K}$ with the property that $h(z, t) \notin \hat{K}$ for $t>0$. Define $H(z, t)=f(z)-h(z, t)$ for $z \in J, t \in[0,1]$, as in the previous paragraph. Then $H$ is a homotopy from $z \rightarrow f(z)-z$ to $z \rightarrow f(z)-\mathrm{c}$ in $\mathbb{C}-\{0\}$. The winding number of $z \rightarrow f(z)-\mathrm{c}$ around 0 is zero, because $\mathrm{c} \notin \hat{K}$. This shows that the winding number of $z \rightarrow f(z)-z$ around 0 is also zero. Therefore index $(f)=0$, in the case where the interiors of $\hat{K}$ and $\hat{J}$ are disjoint.

To prove part (3), it remains to consider the case where $J$ and $K$ intersect in exactly 2 points and neither $J \subset \hat{K}$ nor $K \subset \hat{J}$. In that case, let $h:(J \cup K) \times[0,1] \rightarrow \mathbb{C}$ be an isotopy from the identity map on $J \cup K$ to a map $g: J \cup K \rightarrow \mathbb{C}$ with the property that $g(J)$ is the square and $g(K)$ is the circle indicated in Figure 2.1. Let $H(z, t)=h(f(z), t)-h(z, t)$ for $z \in J, t \in[0,1]$. Then $H$ is a homotopy in $\mathbb{C}-\{0\}$ from $z \rightarrow f(z)-z$ to $z \rightarrow g(f(z))-g(z)$; therefore index $(f)$ is the winding number of $z \rightarrow g(f(z))-g(z)$ around 0 . If $z_{0}$ is some point where $g(f(z))-g(z)$ is real and positive, then $g\left(z_{0}\right)$ must be in the line segment joining the 2 intersection points of $g(J)$ and $g(K)$; obviously $g\left(f\left(z_{0}\right)\right)$ is to the right of this line segment. Therefore, as $z$ moves near $z_{0}$ in the positive direction along $J$, the imaginary part of $g(z)$ decreases and the imaginary part of $g(f(z))$ increases. This implies that the imaginary part of $g(f(z))-g(z)$ is increasing near every point $z_{0} \in J$ where the curve $z \rightarrow g(f(z))-g(z)$ crosses the positive real ray. Thus the winding number around 0 of this curve is nonnegative, and the proof of (3) is complete.

Now part (4) follows from (2) and (3).

We now recall another well-known fact. 


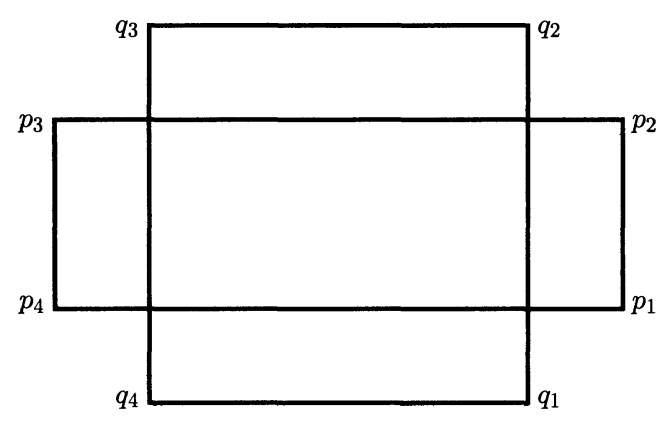

Figure 2.2. These rectangles are conformally unequivalent.

FACT 2.3. Let $\Omega \subset \mathbb{C}$ be open and connected and let $f: \Omega \rightarrow \mathbb{C}$ be an analytic map that is not the identity. Then $f$ has only isolated fixed points in $\Omega$; and if $z$ is a fixed point of $f$, then $\operatorname{index}(f, z) \geqslant 1$.

Proof. Since $f$ is not the identity, $f(z)-z$ is not identically 0 and therefore has only isolated zeroes in $\Omega$. So the fixed points of $f$ are isolated. The multiplicity of a 0 of $f(z)-z$ is the fixed-point index of $f$ at that point. Thus index $(f, z) \geqslant 1$ at each fixed point $z$.

Example. Consider the two rectangles in Figure 2.2. Let $f$ be a homeomorphism between them that maps $p_{1}, p_{2}, p_{3}, p_{4}$ to $q_{1}, q_{2}, q_{3}, q_{4}$, respectively. Then it is easy to see that the fixed-point index of $f$ is -1 . One concludes that there is no conformal homeomorphism between these rectangles whose continuous extension to the boundary takes each $p_{i}$ to $q_{i}$; that is, the quadrilaterals have distinct conformal moduli. This result is hardly surprising, but it is meant to exhibit a simple application of the fixed-point index, which is very much in the spirit of our arguments below.

The next corollary, which is more or less immediate from the above observations, will be most useful.

CoROLlaRY 2.4. Let $f: \Omega \rightarrow \Omega^{*}$ be a conformal homeomorphism between bounded plane domains that extends continuously to a homeomorphism $F: \bar{\Omega} \rightarrow \bar{\Omega}^{*}$. Let $B_{0} \subset B(\Omega)$ be a finite collection of boundary components of $\Omega$. Suppose that the following conditions hold:

(1) $\Omega$ has at most countably many boundary components;

(2) all of the boundary components in $B(\Omega)-B_{0}$ and $B\left(\Omega^{*}\right)-f^{B}\left(B_{0}\right)$ are circles and points, and all of the boundary components in $B_{0}$ are Jordan curves;

(3) $B_{0}$ contains the boundary component of $\Omega$ that is contained in the unbounded component of $\mathbb{C}-\Omega$; and similarly, $f^{B}\left(B_{0}\right)$ contains the boundary component of $\Omega^{*}$ that is contained in the unbounded component of $\mathbb{C}-\Omega^{*}$; 
(4) $F$ has no fixed points in any of the boundary components in $B_{0}$. Let $n$ be the index of the restriction of $F$ to the boundary components in $B_{0}$. Then $f$ has at most $n$ fixed points in $\Omega$. Furthermore, if $S$ is a set of fixed points of $f$, then the total number of fixed points for $f$ in $S$, counting multiplicity, is at most $n$.

Note that conditions (4) and (3) imply that $f$ is not the identity and therefore, being conformal, has only isolated fixed points in $\Omega$. One conclusion of the corollary is that $n \geqslant 0$.

Proof. Let $S$ be a finite set of fixed points of $f$ and let $m$ be the total number of fixed points in $S$, counting multiplicity. Because $f$ is conformal, all of the fixed points of $f$ have positive multiplicity. Therefore it is sufficient to show that $m \leqslant n$.

Our first goal is to make a perturbation to the case that $F$ has no fixed points in $\partial \Omega$. For any constant $\mathrm{c} \in \mathbb{C}$ with $|\mathrm{c}|$ sufficiently small, the map $z \rightarrow F(z)+\mathrm{c}$ will have at least $m$ fixed points, counting multiplicities, in some neighborhood of $S$. (This follows from Rouché's theorem, and it is also clear from the topological definition.)

Let $J \in B(\Omega)-B_{0}$ be a boundary component of $\Omega$. Since $J$ and $f^{B}(J)$ are circles or points, the set of complex numbers c such that the mapping $z \rightarrow F(z)+\mathrm{c}$ has a fixed point in $J$ is a closed set with empty interior in $\mathbb{C}$. Therefore, since the collection of boundary components is countable, by Baire category considerations there are complex numbers $\mathrm{c}$ arbitrarily close to 0 so that $z \rightarrow F(z)+\mathrm{c}$ has no fixed points on the boundary components in $B(\Omega)-$ $B_{0}$. Since $F$ has no fixed points on the boundary components in $B_{0}$, we can find a $\mathrm{c} \in \mathbb{C}$ arbitrarily close to 0 such that $z \rightarrow F(z)+\mathrm{c}$ will have no fixed points in $\partial \Omega$. Pick such a $\mathrm{c}$ with $|\mathrm{c}|$ sufficiently small that

(a) $z \rightarrow f(z)+\mathrm{c}$ has at least $m$ fixed points in $\Omega$, counting multiplicity,

(b) the index of the restriction of the map $z \rightarrow F(z)+\mathrm{c}$ to the boundary components in $B_{0}$ is still $n$. Define $F_{\mathrm{c}}(z)=F(z)+\mathrm{c}$.

If $\left\{z_{0}\right\}$ is a boundary component of $\Omega$ consisting of a single point, then $F_{\mathrm{c}}\left(z_{0}\right) \neq z_{0}$, since $F_{\mathrm{c}}$ has no fixed points in $\partial \Omega$.

When $J$ is a circle in $\mathbb{C}$, we will denote by $D(J)$ the closed disk in $\mathbb{C}$ determined by $J$. Let $B_{+}$be the set of boundary components $J$ in $B(\Omega)-B_{0}$, which are circles and for which $D(J) \cap D\left(F_{\mathrm{c}}(J)\right) \neq \emptyset$. Let $B_{-}$consist of all the other circle boundary components in $B(\Omega)-B_{0}$.

The set $B_{+}$is necessarily finite. To see this, consider an infinite sequence of distinct circles in $B_{+}$. The radii of these circles must tend to 0 . By taking a subsequence, if necessary, we may assume that this sequence of circles converges to some point. This point would necessarily be a fixed point for $F_{\mathrm{c}}$ on $\partial \Omega$, which gives a contradiction and shows that $B_{+}$is finite. 
Let $\Sigma=\bar{\Omega} \cup\left(\bigcup_{J \in B_{-}} D(J)\right)$. We now continuously extend the map $F_{\mathrm{c}}$ to a map $G: \Sigma \rightarrow \mathbb{C}$ by letting the restriction of $G$ to every $D(J), J \in B_{-}$, be an arbitrary homeomorphism onto $D\left(F_{\mathrm{c}}(J)\right)$, which agrees with $F_{\mathrm{c}}$ on $J$. By the definition of $B_{-}$, the fixed points of $G$ will be exactly those of $F_{c}$.

Since $B_{+}$is finite, $\Sigma$ is a closed set, whose boundary consists of finitely many Jordan curves $\left(B_{0} \cup B_{+}\right)$. What is the index of the restriction of $G$ to $\partial \Sigma$ ? The index of the restriction of $G$ to the boundary components in $B_{0}$ is $n$. On the other hand, the Circle Index Lemma 2.2 tells us that the index of the restriction of $G$ to every $J \in B_{+}$is nonnegative, since $J$ and $G(J)$ are circles, provided that we consider $J$ with the orientation induced by $D(J)$ and that $G$ is orientation preserving. But for $J \in B_{+}$the disks $D(J)$ and $D(G(J))$ are disjoint from $\Sigma$ and $G(\Sigma)$, respectively; hence $G$ is orientation preserving, and the orientation we must consider for $J$ is the opposite orientation from the one induced by $D(J)$. Therefore the index of the restriction of $G$ to every $J \in B_{+}$ is nonpositive. Thus the index of the restriction of $G$ to $\partial \Sigma$ is at most $n$.

We now appeal to Theorem 2.1, and conclude that the total number of fixed points of $G$, counting multiplicities, is at most $n$. Certainly the same would be true for $F_{\mathrm{c}}$. This gives $n \geqslant m$, since every fixed point of $G$ has positive multiplicity, and so the proof of the corollary is complete.

\section{The Uniqueness Theorem}

Recall that a circle domain in $\hat{\mathbb{C}}$ is a connected open subset of $\hat{\mathbb{C}}$, all of whose boundary components are circles and points. We now restate the uniqueness part of Theorem 0.1 .

The Uniqueness Theorem 3.1. Let $\Omega, \Omega^{*}$ be circle domains in the Riemann sphere having at most countably many boundary components. Suppose that $f: \Omega \rightarrow \Omega^{*}$ is a conformal homeomorphism of $\Omega$ onto $\Omega^{*}$. Then $f$ is a restriction to $\Omega$ of a Möbius transformation and, in particular, $\Omega$ and $\Omega^{*}$ are Möbius equivalent.

Boundary EXTENsion TheOREM 3.2. Let $\Omega, \Omega^{*}$ be open connected sets in the Riemann sphere and let $f: \Omega \rightarrow \Omega^{*}$ be a conformal homeomorphism between them. Let $W$ be an open subset of $B(\Omega)$, which is at most countable. Suppose that the boundary components of $\Omega$ corresponding to elements of $W$ are all circles and points and that the corresponding (under $f$ ) boundary components of $\Omega^{*}$ are also all circles and points. Then $f$ extends continuously to the boundary components in $W$ and extends to a homeomorphism between $\bigcup\{K: K \in W\} \cup \Omega$ and $\bigcup\left\{K^{*}: K^{*} \in f^{B}(W)\right\} \cup \Omega^{*}$.

In the hypotheses of the theorem, we do not assume that $f^{B}(K)$ is a circle when $K \in W$ is a circle or that $f^{B}(K)$ is a point when $K \in W$ is a point. 
The proof of Theorem 3.2 will appear in the following sections. Now we will see how the Uniqueness Theorem 3.1 follows from our Boundary Extension Theorem 3.2. This was also pointed out by Strebel [Str1] (who was unable to prove Theorem 3.2).

Proof of the Uniqueness Theorem 3.1. Assuming Theorem 3.2, we apply it with $W=B(\Omega)$ to conclude that $f$ extends continuously to a homeomorphism $F$ from $\bar{\Omega}$ to $\bar{\Omega}^{*}$ (closures in $\hat{\mathbb{C}}$ ).

Since we are free to normalize in the domain and range by Möbius transformations, we assume, without loss of generality, that $\infty \in \Omega$ is a fixed point for $f$ and that $f$ has the form

$$
f(z)=z+\frac{a_{1}}{z}+\frac{a_{2}}{z^{2}}+\cdots
$$

near $\infty$. If all of the coefficients $a_{j}$ are 0 , then $f(z)=z$, and we are done. Suppose that $j$ is the least positive integer with $a_{j} \neq 0$. Then there is a real number $R$ large enough that $\partial D(0, R) \subset \Omega$ and $\left|f(z)-z-a_{j} z^{-j}\right|<\left|a_{j} z^{-j}\right|$ for $z \in \partial D(0, R)$, where $D(0, R)$ is the disk centered at 0 with radius $R$. The index of the restriction of $f$ to $\partial D(0, R)$ is the same as the winding number around 0 of the restriction of $z \rightarrow a_{j} z^{-j}$ to $\partial D(0, R)$, because the homotopy $H: \partial D(0, R) \times[0,1] \rightarrow \mathbb{C}, H(z, t)=(1-t)(f(z)-z)+t a_{j} z^{-j}$, has no zeroes. But that winding number is $-j$, which is negative. If we now look at the restriction of $f$ to $\Omega \cap D(0, R)$, this gives a contradiction to Corollary 2.4 with $B_{0}=\{\partial D(0, R)\}$. This contradiction shows that all of the coefficients $a_{j}$ are 0 , completing the proof.

\section{The Schwarz-Pick lemma}

The generalization Theorem 0.6 of the Schwarz-Pick lemma is of central importance in this work; ${ }^{3}$ we now start its proof.

Note that the assumptions in Theorem 0.6 imply that $\Omega$ is a domain that has at most countably many boundary components, and all but one of them are circles and points. The same holds for $\Omega^{*}$.

The following lemma, which will also be used in the proof of Theorem 3.2, shows that Theorem 0.6 reduces to Theorem 3.2.

LEMma 4.1. Theorem 0.6 is true under the additional hypothesis that $f$ extends to a homeomorphism of $\bar{\Omega}-\partial A$ onto $\bar{\Omega}^{*}-\partial A^{*}$.

Proof. Let $p \neq q$ be two points in $\Omega$ and assume first that $f(p)=p$ and $f(q)=q$. Suppose that $f$ is not the identity. Let $h(z)=a z$, where

\footnotetext{
${ }^{3} \mathrm{~A}$ generalization of this Schwarz-Pick lemma for noninjective mappings will appear in a subsequent paper.
} 
$|a|<1$, but $a$ is sufficiently close to 1 that the composition $h \circ f$ still has 2 distinct fixed points, $p^{\prime}$ near $p$ and $q^{\prime}$ near $q$. Now $h\left(\bar{A}^{*}\right) \subset U$. Therefore there is a Jordan curve $\gamma \subset \Omega$, which separates $h\left(\Omega^{*}\right)$ from $\partial A$. Since $h \circ f$ maps $\gamma$ into $h\left(\Omega^{*}\right)$, the index of the restriction of $h \circ f$ to $\gamma$ is 1, by part (2) of Lemma 2.2. Let $\Sigma$ be the intersection of $\Omega$ with the Jordan domain bounded by $\gamma$. The points $p^{\prime}$ and $q^{\prime}$ must certainly be in $\Sigma$, because they are in $\Omega \cap h\left(\Omega^{*}\right)$. We therefore get a contradiction to Corollary 2.4 by considering the restriction of $h \circ f$ to $\Sigma$ and taking $B_{0}=\{\gamma\}$. This contradiction shows that $f$ must be the identity if it fixes 2 distinct points.

We now deal with the general case. Suppose that $p, q \in \Omega \cap U, p \neq q$, and $d_{\text {hyp }}(f(p), f(q)) \geqslant d_{\text {hyp }}(p, q)$. Then there is a Möbius transformation $g$, which takes $f(p)$ to $p$, takes $f(q)$ to $q$ and takes $U$ into $U$. (If $g_{1}$, and $g_{2}$ are hyperbolic isometries of $U$ taking $p$ and $f(p)$, respectively, to 0 , then one can take $g(z)=g_{1}^{-1}\left(g_{2}(z) g_{1}(q) / g_{2}(f(q))\right)$.) Then, by the above, the composition $g \circ f$ is the identity on $\Omega^{*}$. Now $g$, being the inverse of $f$, maps $A^{*} \subset U$ onto $A \supset U$. Hence $g$ is a Möbius transformation, which maps $U$ onto $U$, and it is therefore a hyperbolic isometry. This implies that $f$ is the restriction of a hyperbolic isometry and completes the proof of the lemma.

\section{Extension to the boundary}

In this section we will prove Theorem 3.2 using transfinite induction. In the inductive step we will encounter the situation where a conformal map $f$ extends continuously to all but perhaps one of the boundary components of its domain, and we will have to prove that it also continuously extends to that one boundary component. The following lemmas, like Lemma 4.1, deal with the situation where there is only one boundary component to which we do not know if $f$ extends.

Lemma 5.1. Let $f: \Omega \rightarrow \Omega^{*}$ be a conformal homeomorphism between connected open subsets of the unit disk $U$, which extends continuously to a homeomorphism from $\bar{\Omega}-\partial U$ onto $\bar{\Omega}^{*}-\partial U$. Suppose that $\partial U$ is a boundary component of both $\Omega$ and $\Omega^{*}$ and that $f^{B}(\partial U)=\partial U$. Further suppose that $J$ and $J^{*}=f^{B}(J)$ are corresponding boundary components of $\Omega$ and $\Omega^{*}$, all other boundary components of $\Omega$ and $\Omega^{*}$ are circles and points and there are at most countably many such boundary components. Consider $U$ with the hyperbolic metric. Then, given any $\epsilon>0, f$ is bi-Lipschitz (in the hyperbolic metric) on the set $L_{\epsilon}$ of points in $\Omega$ having hyperbolic distance $>\epsilon$ from $J$. Furthermore the restriction of $f$ to $L_{\epsilon}$ extends to a bi-Lipschitz homeomorphism from $U$ onto $U$. 
Proof. We will first verify a Lipschitz condition near 0 . Assume that $J$ does not separate 0 from $\Omega$. Let $a>0$ be some number less than the euclidean distance from 0 to $J$ and set $g(z)=z / a$. Then $g(J)$ does not intersect $\bar{U}$. Let $\gamma \subset g(\Omega)$ be some Jordan curve separating $g(J)$ from $U$ and let $\Sigma$ be the connected component of $g(\Omega)-\gamma$ containing 0 . The restriction of the map $f \circ g^{-1}$ to $\Sigma$ then satisfies the conditions of Lemma 4.1. We conclude from that lemma that $f \circ g^{-1}$ is hyperbolic-length-decreasing on $\Sigma \cap U$. But the restriction of $g$ to the disk $D(0, a / 2)$ of euclidean radius $a / 2$ around 0 is Lipschitz in the hyperbolic metric, with the Lipschitz constant $l=l(a)$ depending only on $a$. Since $f \circ g^{-1}$ is contracting, it follows that $f$ is Lipschitz with constant $l(a)$ on $D(0, a / 2) \cap \Omega$.

For every number $t \in(0,1)$ let $h(t)$ denote the hyperbolic radius of a circle of euclidean radius $t$ around 0 . Since we may precompose $f$ by any hyperbolic isometry, our above Lipschitz condition near 0 translates to every point in $U-J$ as follows: Let $p$ be a point in $U$ whose hyperbolic distance from $J$ is greater than $h(a)$; then $f$ has Lipschitz constant $l(a)$ on $\Omega \cap D_{\text {hyp }}(p, h(a / 2))$, where $D_{\text {hyp }}(p, r)$ denotes the disk of hyperbolic radius $r$ centered at $p$. By taking limits, we find that the continuous extension of $f$ to $\bar{\Omega}-\partial U$, which we continue to denote by $f$, is Lipschitz with constant $l(a)$ on $\bar{\Omega} \cap D_{\text {hyp }}(p, h(a / 2))$.

Let $M \notin\{J, \partial U\}$ be some circle boundary component of $\Omega$, which bounds a disk $D(M)$, and let $M^{*}$ be the corresponding boundary component of $\Omega^{*}$. As we have seen, for every point $p$ in $M, f$ satisfies a local Lipschitz condition at $p$. (The local Lipschitz constant of $f$ at $p$ is, by definition,

$$
\lim \sup d_{\text {hyp }}\left(f\left(p_{1}\right), f\left(p_{2}\right)\right) / d_{\text {hyp }}\left(p_{1}, p_{2}\right)
$$

as $p_{1}$ and $p_{2}$ tend to $p$, while $p_{1} \neq p_{2}$.) But one can further extend $f$ to $D(M)$ by mapping the hyperbolic center of $D(M)$ to the center of the corresponding disk $D\left(M^{*}\right)$ and extending radially. Clearly the local Lipschitz constant of this extension at any point in $D(M)$ is at most the supremum of the local Lipschitz constants of $f$ in $M$. We extend $f$ in this way over the interior of every such boundary circle $M$ to obtain a map $F$.

Now take any $\epsilon^{\prime}>0$ and let $R_{\epsilon^{\prime}}$ be the set of points in $U$ having distance of at least $\epsilon^{\prime}$ from $J$ and which are not separated from $\partial U$ by $J$. From the above, it follows that $F$ satisfies a uniform local Lipschitz condition on $R_{\epsilon^{\prime}}$; that is, there is some constant $l$ such that all of the local Lipschitz constants of $F$ in $R_{\epsilon^{\prime}}$ are bounded by $l$. Since the same arguments can be applied to the inverses of $f$ and $F$, and since $F\left(R_{\epsilon^{\prime}}\right)$ is bounded away from $J^{*}$, we conclude that $F^{-1}$ also satisfies a uniform local Lipschitz condition on $F\left(R_{\epsilon^{\prime}}\right)$. It is easy to see that one can modify $F$ in the complement of $R_{\epsilon}$ and then extend $F$ to the whole of $U$ so that the resulting homeomorphism, which we will continue to denote by $F$, as well as its inverse will satisfy a uniform local 
Lipschitz condition on $U$. (To get an explicit construction take an analytic Jordan curve $\beta \subset \Omega$, which separates $J$ from $R_{\epsilon}$. Let $D_{1}$ be the topological disk bounded by $\beta$, let $D_{2}$ be the topological disk bounded by $F(\beta)$ and let $g_{1}$ and $g_{2}$ be Riemann maps from a euclidean geometric disk $D$ to $D_{1}$ and $D_{2}$, respectively. Note that $g_{1}$ and $g_{2}$ extend analytically to $\partial D$. Consider the map $g: \partial D \rightarrow \partial D$ defined by $g(z)=g_{2}^{-1}\left(F\left(g_{1}(z)\right)\right)$. This map $g$ is analytic and therefore bi-Lipschitz in $\partial D$. Let $G$ be the radial extension of $g$ to $D$. Then $G$ is bi-Lipschitz. Let $F$ stay as it is outside $D_{1}$ and, in $D_{1}$, redefine $F$ by $F(z)=g_{2}\left(G\left(g_{1}^{-1}(z)\right)\right)$. In other words, with $g_{1}$ and $g_{2}$ parametrize $\bar{D}_{1}$ and $\bar{D}_{2}$ as euclidean disks and then use the usual euclidean radial extension. Because $g_{1}, g_{2}$ and $G$ are bi-Lipschitz, so is the restriction of $F$ to $\bar{D}_{1}$.)

Since the domain of $F$ is now $U$, which is hyperbolically convex, clearly $F$ is bi-Lipschitz (globally). (If $p, q \in U$, take the hyperbolic line segment joining $p$ and $q$; the image of that segment will be a path of length at most $d_{\text {hyp }}(p, q) l$ joining $F(p)$ and $F(q)$, where $l$ is the uniform local Lipschitz constant of $F$. This gives $d_{\text {hyp }}(F(p), F(q)) \leqslant l d_{\text {hyp }}(p, q)$. A similar argument with $F^{-1}$ gives $l^{\prime} d_{\text {hyp }}(F(p), F(q)) \geqslant d_{\text {hyp }}(p, q)$.) Since $L_{\epsilon} \subset R_{\epsilon}$, this completes the proof of Lemma 5.1.

Remark. Though we will not use this, the above argument can be used to show that the Lipschitz constant of $f$ on the set $L_{d}$ tends to 1 as $d \rightarrow \infty$.

Lemma 5.2. Let $f: \Omega \rightarrow \Omega^{*}$ be a conformal homeomorphism between open connected subsets of $\hat{\mathbb{C}}$. Let $J \neq K$ be boundary components of $\Omega$ and let $J^{*}$ and $K^{*}$ be the corresponding boundary components of $\Omega^{*}$. Suppose that $\Omega$ has at most countably many boundary components and all of them, with the possible exception of $J$, are circles and points. Similarly assume that all of the boundary components of $\Omega^{*}$, with the possible exception of $J^{*}$, are circles and points. Also assume that $f$ extends continuously to a homeomorphism of $\bar{\Omega}-K$ onto $\bar{\Omega}^{*}-K^{*}$. Then $K$ is a circle if and only if $K^{*}$ is a circle.

Proof. We assume that $K$ is a circle and $K^{*}$ is a point. Since the situation is symmetric, it is enough to reach a contradiction in this case. Since $K^{*}$ is a point, $f$ extends continuously to $K$ and maps $K$ to $K^{*}$. By replacing $J$ with some Jordan curve in $\Omega$ separating $J$ from $K$, we assume, without loss of generality, that $J$ and $J^{*}$ are Jordan curves.

Normalizing with Möbius transformations allows us to assume that the situation is as in Figure 5.1; that is, $K^{*}=\{0\} ; K$ is a circle with center 0 which separates 0 from $J ; J$ separates $K$ from $J^{*}$; and $J^{*}$ separates $J$ from $\infty$. By further renormalization we want to replace $f$ with a map which fixes some point in $\Omega$, without losing the above properties. To achieve that let $a=\max \{|z|: z \in J\}$, let $r$ be the (euclidean) radius of $K$ and let $d$ 


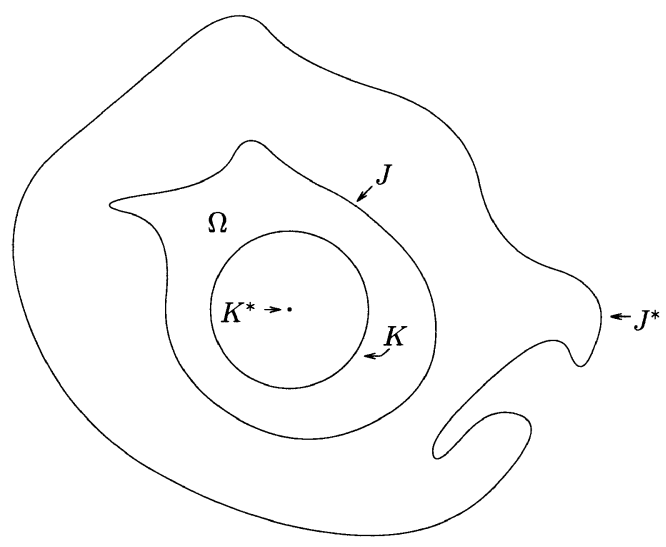

FiguRE 5.1

be the (euclidean) distance from 0 to $J^{*}$. Pick some point $p \in \Omega$, so that $|f(p)|<r d / a$, and consider the map $g(z)=f(z) p / f(p)$. We have $g(p)=p$. Since $|p|>r$, it follows that $|p / f(p)|>a / d$ and, therefore, $g^{B}(J)$ is disjoint from $J$ and separates $J$ from $\infty$. Let $F: \bar{\Omega} \rightarrow \overline{g(\Omega)}$ be the continuous extension of $g$. By the Circle Index Lemma 2.2, the index of the restriction of $F$ to $J$ is 1 and the index of the restriction of $F$ to $K$ is -1 , when $K$ has the orientation induced by $\Omega$; the sum of these is 0 . But $F$ has a fixed point at $p$, which contradicts Corollary 2.4 with $B_{0}=\{K, J\}$. This completes the proof of Lemma 5.2.

Proof of Theorem 3.2. We will prove by transfinite induction on $\alpha$ that, for each countable ordinal $\alpha$, the map $f$ extends continuously to a homeomorphism from $\Omega \cup\left(\bigcup_{K \in W_{\alpha}} K\right)$ to $\Omega^{*} \cup\left(\bigcup_{K^{*} \in W_{\alpha}^{*}} K^{*}\right)$, where $W_{\alpha}$ is the collection of boundary components in $W$ having rank at most $\alpha$ and $W_{\alpha}^{*}$ is the collection of corresponding boundary components of $\Omega^{*}$.

Suppose that this holds for all ordinals $\beta<\alpha$. Let $K \in W$ be a boundary component of rank $\alpha$ and let $K^{*}$ be the corresponding boundary component of $\Omega^{*}$. Let $J \subset \Omega$ be a Jordan curve separating $K$ from every other boundary component of $\Omega$ that has rank $\geqslant \alpha$, and from every boundary component outside $W$. And let $\Sigma$ be the connected component of $\Omega-J$, which has $K$ as a boundary component. Denote by $\Sigma^{*}$ the image of $\Sigma$ under $f, \Sigma^{*}=f(\Sigma)$, and let $F$ denote the restriction of $f$ to $\Sigma$. We shall show that $F$ extends to a homeomorphism from $\bar{\Sigma}$ onto $\bar{\Sigma}^{*}$. This will clearly suffice to complete the inductive step.

By the inductive hypothesis we already know that $F$ extends to a homeomorphism from $\bar{\Sigma}-K$ onto $\bar{\Sigma}^{*}-K^{*}$. Lemma 5.2 shows that $K$ and $K^{*}$ are either both points or both circles. If $K$ and $K^{*}$ are points, then it is obvious 
that $F$ extends as needed, and we only consider the case where $K$ and $K^{*}$ are circles. Since we are free to renormalize by Möbius transformations, we assume, without loss of generality, that $K=K^{*}=\partial U$ and that $\Sigma, \Sigma^{*} \subset U$. Now Lemma 5.1 applies and shows that the restriction of $F$ to the set of points having hyperbolic distance at least 1 , say, from $J$ extends to a bi-Lipschitz homeomorphism $g: U \rightarrow U$. We briefly reproduce here, for the convenience of the reader, a standard geometric argument (maybe due to Mostow), which shows that $g$ extends to a self-homeomorphism of $\bar{U}$. (Alternatively one can conclude that $g$ extends to a homeomorphism of $\bar{U}$ from the fact that $g$ is quasiconformal.)

Let $l$ be the bi-Lipschitz constant of $g$. Consider some straight ray $A$ of infinite hyperbolic length starting at 0 . We now show that $g(A)$ has 1 limit point in $\partial U$. Take some $r>0$. Since the diameter of a disk of radius $r+2$ is $2(r+2)$, the preimage under $g$ of the disk with center the origin and radius $r+2$ has diameter at most $2(r+2) l$. Therefore the total length of the part of $g(A)$, whose distance from 0 is between $r$ and $r+2$, is at most $2(r+2) l^{2}$. This implies that

$$
\sum_{E \in H(r)} \theta(E) \leqslant 2 \pi \frac{2(r+2) l^{2}}{p(r)},
$$

where $H(r)$ denotes the collection of connected components of the intersection of $g(A)$ with the open annulus between the circles of radii $r$ and $r+2$ around 0 , and where each $\theta(E)$ denotes the angular diameter of $E$ with respect to $0, \theta(E)=\sup _{x, y \in E} \angle(x, 0, y)$, and $p(r)$ is the length of the perimeter of a hyperbolic circle with radius $r$. Since $p(r)$ increases exponentially as $r \rightarrow \infty$, it follows that $\lim _{n \rightarrow \infty} \sum_{r=n}^{\infty} \sum_{E \in H(r)} \theta(E)=0$. This shows that $g(A)$, which clearly has some limit points in $\partial U$, has in fact a unique limit point there. Now we extend $g$ to $\partial U$ by letting $g(p)$ be the unique limit point of the ray $g(A)$, where $A$ is the ray $[0, p)$ and $p$ is any point in $\partial U$. One easily uses the above inequalities to verify that $g$ extended thusly is continuous. Checking that it is a homeomorphism is also straightforward.

This clearly implies that $f$ extends as needed, completing the inductive step. An appeal to the principle of transfinite induction now establishes the theorem. (Note that it is not necessary to verify the base of the induction, since the inductive hypothesis is empty when $\alpha=0$. Of course the base of the induction is also standard.)

\section{Maximum modulus, normality and angles}

The results of this section, besides their independent interest, will prepare us for the proof of the existence part of Theorem 0.1. 
Maximum Modulus Theorem 6.1. Let $A$ and $A^{*}$ be Jordan domains in $\mathbb{C}$; let $\Omega$ be a domain which is obtained from $A$ by the deletion of a closed disjoint union of at most countably many closed (geometric) disks and points in $A$; and similarly let $\Omega^{*}$ be a domain obtained from $A^{*}$ by the deletion of a closed disjoint union of at most countably many closed disks and points. Suppose that $f: \Omega \rightarrow \Omega^{*}$ is a conformal homeomorphism between $\Omega$ and $\Omega^{*}$ that extends continuously to $\partial A$, and that $f^{B}(\partial A)=\partial A^{*}$. Then

$$
f(z)-z \in \text { convex hull }\{f(w)-w: w \in \partial A\}
$$

for every point $z \in \Omega$. In particular,

$$
\sup _{z \in \Omega}|f(z)-z|=\max _{w \in \partial A}|f(w)-w| .
$$

Proof. Note first that Theorem 3.2 implies that $f$ extends to a homeomorphism between the closures of $\Omega$ and $\Omega^{*}$.

Let $z_{0} \in \Omega$ and define $g(z)=f(z)-f\left(z_{0}\right)+z_{0}$. Then $z_{0}$ is a fixed point for $g$. Assume that $f\left(z_{0}\right)-z_{0}$ is not in the convex hull of $\{f(w)-w: w \in \partial A\}$. It then follows that 0 is not in the convex hull of $\{g(w)-w: w \in \partial A\}$. Therefore the winding number around 0 of the restriction of $w \rightarrow g(w)-w$ to $\partial A$ is 0 . This means that the restriction of $g$ to $\partial A$ has index 0 . However $g$ has a fixed point at $z_{0}$, in contradiction to Corollary 2.4. This proves the first assertion, and the second assertion clearly follows.

By the same method as above, it is possible to get estimates analogous to Cauchy's estimates for the first and second derivative. This will be done in a subsequent paper.

CoROLlaRY 6.2 (Normality). Let $\Omega$ be as above and let $f_{k}: \Omega \rightarrow \Omega_{k}^{*}$ be a sequence of conformal homeomorphisms such that each $f_{k}$ and $\Omega_{k}^{*}$ satisfy the conditions placed on $f$ and $\Omega^{*}$ above. Suppose that the $f_{k}$ converge uniformly on compact subsets of $\Omega$ to a function $g$. Then $g$ is either a constant map or a conformal homeomorphism, and $g^{B}(K)$ is a circle or a point for every boundary component $K \in B(\Omega)-\{\partial A\}$. Moreover the convergence to $g$ is uniform on any subset of $\Omega$ whose closure does not intersect $\partial A$.

Proof. We start with the last assertion. Let $E$ be some subset of $\Omega$ whose closure does not intersect $\partial A$, and let $\epsilon>0$. There is some Jordan curve $\gamma \subset \Omega$ separating $E$ from $\partial A$. The convergence on $\gamma$ is uniform. Therefore there is some integer $N$ so that $\left|f_{m}(z)-f_{k}(z)\right|<\epsilon$ for all $k, m>N$ and all $z \in \gamma$; equivalently, $\left|f_{m} \circ f_{k}^{-1}(w)-w\right|<\epsilon$ for $k, m>N, w \in f_{k}(\gamma)$. Now apply the Maximum Modulus Theorem 6.1 to the maps $f_{m} \circ f_{k}^{-1}$ to conclude that $\left|f_{m} \circ f_{k}^{-1}(w)-w\right|<\epsilon$ for all $k, m>N$ and all $w \in f_{k}(E)$. This gives 
$\left|f_{m}(z)-f_{k}(z)\right|<\epsilon$, for all $k, m>N$ and all $z \in E$, and implies the uniform convergence.

That $g$ is either a constant or a conformal homeomorphism is a well-known consequence of Rouché's theorem. Let $K \in B(\Omega)-\{\partial A\}$, let $W$ be an open set that contains $K$ and whose closure is disjoint from $\partial A$, and set $W_{0}=W \cap \Omega$. By the above, the convergence $f_{k} \rightarrow g$ is uniform on $W_{0}$. Let $\epsilon>0$ and let $k$ be large enough that $\left|f_{k}(z)-g(z)\right|<\epsilon$ for $z \in W_{0}$. Then clearly the distance from any point in $g^{B}(K)$ to $f_{k}^{B}(K)$ is at most $\epsilon$, and the distance from any point in $f_{k}^{B}(K)$ to $g^{B}(K)$ is at most $\epsilon$. In other words, the Hausdorff distance from $g^{B}(K)$ to $f_{k}^{B}(K)$ is at most $\epsilon$. Since $f_{k}^{B}(K)$ is a circle or a point, and since $\epsilon$ was arbitrary, we conclude that $g^{B}(K)$ is a circle or a point, because the collection of circles and points is closed in the Hausdorff metric. This completes the proof of the normality corollary.

Definition 6.3. Let $\eta \subset \hat{\mathbb{C}}$ be a circular arc with endpoints $p, q$ and let $z$ be some point not on the circle containing $\eta$. We define the angle of $\eta$ from $z$, denoted by $\operatorname{ang}(z, \eta)$, to be the length of $m(\eta)$, where $m$ is any Möbius transformation taking $z$ to 0 and $\eta$ into $\partial U$, the unit circle. (This is the same as the angle at $z$ between the two circular arcs that join $z$ to $p$ and $q$, respectively, and which are orthogonal to $\eta$.)

The definition is clearly Möbius invariant.

ANGLe Lemma 6.4. Let $\Omega$ be a domain obtained from a Jordan domain $A \subset \hat{\mathbb{C}}$ by the removal of a closed, disjoint, countable union of disks and points in $A$. Suppose that $f$ is a conformal homeomorphism from $\Omega$ onto a circle domain that extends continuously to a homeomorphism from $\partial A$ to a circle. Further suppose that $z_{0} \in \Omega$ and $D$ is an open geometric disk containing $z_{0}$ such that the boundary of the connected component of $D \cap A$ that contains $z_{0}$, is the union of the arcs $\alpha \subset \partial A \cap D$ and $\beta \subset \partial D \cap A$, as in Figure 6.1. Then

$$
\operatorname{ang}\left(f\left(z_{0}\right), f(\alpha)\right) \geqslant \operatorname{ang}\left(z_{0}, \eta\right),
$$

where $\eta$ is the arc of $\partial D$ complementary to $\beta$.

In a slight abuse of notation we are using $f$ to denote also the continuous extension of $f$ to $\partial A$.

Proof. By normalizing with Möbius transformations, we assume without loss of generality that $f$ maps $\partial A$ onto $\partial D$, respecting orientation, and that $D=U, z_{0}=f\left(z_{0}\right)$ and $\infty \in \Omega$. Striving for a contradiction, we assume that $\operatorname{ang}\left(f\left(z_{0}\right), f(\alpha)\right)<\operatorname{ang}\left(z_{0}, \eta\right)$, which is equivalent to $\operatorname{ang}\left(f\left(z_{0}\right), f(\alpha)\right)+$ $\operatorname{ang}\left(z_{0}, \beta\right)<2 \pi$. Then, by further normalizing with a hyperbolic isometry of $U$ that fixes $z_{0}$, we assume that $\beta$ and $f(\alpha)$ are disjoint. 


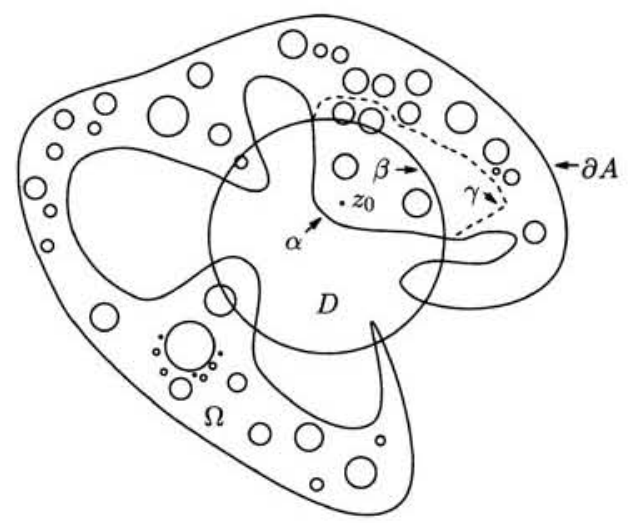

Figure 6.1

Now let $\gamma \subset \Omega-\bar{U}$ be some simple curve with endpoints in $\partial A-U$, such that $\gamma \cup \partial A$ separates $z_{0}$ from any other connected component of $U \cap A$ and from $\infty$. (See Figure 6.1.) It is easy to check that such a $\gamma$ exists. Let $\Omega^{-}$be the connected component of $\Omega-\gamma$ containing $z_{0}$. We now examine the index of the restriction of $f$ to the boundary component $K_{1}$ of $\Omega^{-}$that contains $\gamma$ and $\alpha$. Let $f_{1}$ denote that restriction and let $H: \alpha \times[0,1] \rightarrow U \cup \beta$ be an endpoints-fixing homotopy in $U \cup \beta$ from the identity map on $\alpha$ to some homeomorphism $h_{1}$ from $\alpha$ to $\beta$. Since $f_{1}(\alpha)=f(\alpha) \subset \partial U$ is disjoint from $\beta$, it follows that $H(z, t) \neq f_{1}(z)$ for each $z \in \alpha, t \in[0,1]$. Therefore the index of $f_{1}$ is equal to the index of the map $f_{2}: \beta \cup K_{1}-\alpha \rightarrow \partial U$, defined by $f_{2}(z)=f_{1}(z)$ for $z \in K_{1}-\alpha$ and $f_{2}(z)=f_{1} \circ h_{1}^{-1}(z)$ for $z \in \beta$. But the index of $f_{2}$ is clearly 0 , because the Jordan domain in $\hat{\mathbb{C}}$ determined by $\beta \cup K_{1}-\alpha$ is disjoint from $U$. So the index of $f_{1}$ is 0 .

However, every boundary component $K \in B\left(\Omega^{-}\right)-\left\{K_{1}\right\}$ is a circle or a point, and $f$ maps these to circles and points ( $f$ extends continuously to the boundary, by Theorem 3.2). Since $f\left(z_{0}\right)=z_{0}$ and the index of the restriction $f_{1}$ of $f$ to $K_{1}$ is 0 , this gives a contradiction to Corollary 2.4, as usual. This completes the proof of the lemma.

We will also need the following elementary geometric lemma:

Lemma 6.5. Let $\eta$ be a circular arc with endpoints $p, q$, say. Suppose that $z$ is a point not on the circle containing $\eta$ and let $\delta_{z}$ be the circular arc with endpoints $p, q$ that passes through $z$. Then $\operatorname{ang}(z, \eta)+2 \theta=2 \pi$, where $\theta$ is the angle between $\eta$ and $\delta_{z}$ at $p$ (or at $q$ ).

Proof. By normalizing with a Möbius transformation, we assume that $z=$ $\infty$. Let $o$ be the center of the circle containing $\eta$. Then $\operatorname{ang}(o, \eta)=\operatorname{ang}(z, \eta)$. 


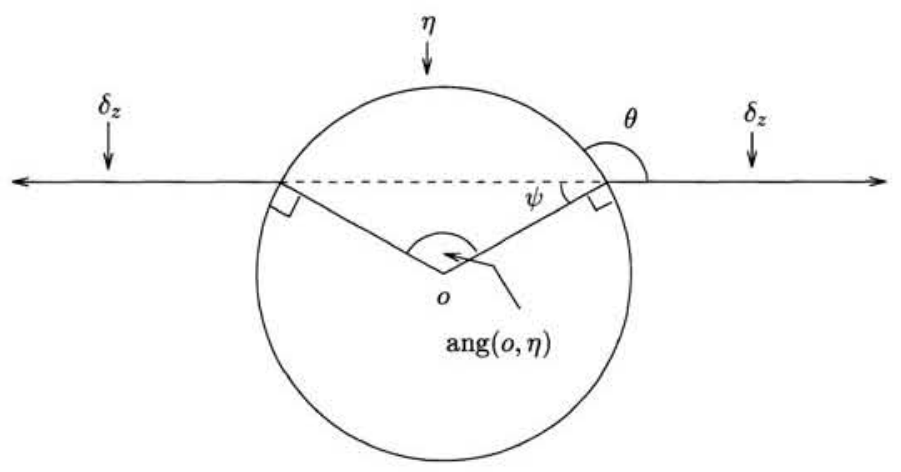

Figure 6.2

Now consider the angle $\psi$ indicated in Figure 6.2. Clearly $\psi+\pi / 2=\theta$ and ang $(o, \eta)+2 \psi=\pi$. From these the lemma follows.

\section{Uniformization}

We will now restate and prove the existence part of Theorem 0.1.

UNIFORMIZATION THEOREM 7.1. Every connected planar domain $\Omega$ with at most countably many boundary components is conformally equivalent to a circle domain.

Proof. The proof will proceed by transfinite induction on the type of $\Omega$. Recall that the type of $\Omega$, denoted by $\operatorname{tp}(\Omega)$, is defined as the pair $(\lambda, n)$ such that $\lambda$ is a countable ordinal, $n$ is a positive integer and $B(\Omega)^{\lambda}$ has $n$ elements. The collection of such pairs is ordered lexicographically; that is, $\left(\lambda_{1}, n_{1}\right)<\left(\lambda_{2}, n_{2}\right)$ if $\lambda_{1}<\lambda_{2}$, or $\lambda_{1}=\lambda_{2}$ and $n_{1}<n_{2}$. Since this is a well ordering, one can transfinitely induct with respect to it.

Let $(\lambda, n)$ be the type of $\Omega$. If $\lambda=0$, that is, if $\Omega$ has finitely many boundary components, then the existence was proved by Koebe. Therefore we will assume that $\Omega$ has infinitely many boundary components and the theorem holds for all domains of lesser type. Let $K_{0}$ be some boundary component of $\Omega$ of rank $\lambda\left(K_{0} \in B(\Omega)^{\lambda}\right)$. Let $J_{k}, k=1,2, \ldots$, be a sequence of Jordan domains satisfying $\partial J_{k} \subset \Omega, J_{k} \subset J_{k+1}$ and $\bigcup_{k=1}^{\infty} J_{k} \supset \bar{\Omega}-K_{0}$. Define $\Omega_{k}=J_{k} \cap \Omega$.

By the inductive hypothesis, since $\operatorname{tp}\left(\Omega_{k}\right)<\operatorname{tp}(\Omega)$, each $\Omega_{k}$ is conformally homeomorphic to some circle domain. For each $k$ let $f_{k}: \Omega_{k} \rightarrow \Omega_{k}^{*}$ be such a homeomorphism. By normalizing with a Möbius transformation, we assume without loss of generality that $f_{k}^{B}\left(\partial J_{k}\right)=\partial U$ and $f_{k}\left(z_{0}\right)=0$, where $z_{0}$ is some arbitrary point in $\Omega_{1}$. Since the sequence $\left\{f_{k}\right\}$ is a normal family, by choosing a subsequence if necessary, we also assume that the maps $f_{k}$ converge 
uniformly on compact subsets of $\Omega$. Let $f$ be the limit of the sequence $\left\{f_{k}\right\}$. Then $f$ is either the constant 0 or a conformal homeomorphism $f: \Omega \rightarrow \Omega^{*}$, where $\Omega^{*} \subset U$. We will consider these two cases separately.

Hyperbolic Case $\left(f \neq\right.$ constant). Since the maps $f_{k}$ converge to $f$ uniformly on compacts of $\Omega$, we conclude from Corollary 6.2 that $f^{B}(K)$ is a circle or a point whenever $K \in B(\Omega)-\left\{K_{0}\right\}$. The application of that corollary is feasible here, because every such $K$ can be separated from $K_{0}$ by a Jordan curve in $\Omega$. It only remains to show that $f^{B}\left(K_{0}\right)=\partial U$.

LEMMA 7.2. Let $W$ be the connected component of $U-f^{B}\left(K_{0}\right)$ which contains $\Omega^{*}=f(\Omega)$. Then $W$ is convex in the hyperbolic metric $d_{\text {hyp }}$ of $U$.

Proof. Let $x, y$ be 2 distinct points in $\Omega$ and let their images under $f$ be $x^{*}, y^{*} \in \Omega^{*}, x^{*}=f(x), y^{*}=f(y)$. Let $\epsilon>0$ and let $m$ be such that $x, y \in \Omega_{m}$, and $d_{\text {hyp }}\left(x^{*}, f_{m}(x)\right)<\epsilon$ and $d_{\text {hyp }}\left(y^{*}, f_{m}(y)\right)<\epsilon$. Let $e$ be the hyperbolic line segment joining $f_{m}(x)$ and $f_{m}(y)$. For $k>m$ now consider the map $h_{k}=f_{k} \circ f_{m}^{-1}$, whose domain is $f_{m}\left(\Omega_{m}\right)$. By the Schwarz-Pick lemma, Theorem 0.6 , it follows that $h_{k}$ is a contraction in the hyperbolic metric and extends to a contraction $\hat{h}_{k}$ of $U$. Define $e_{k}=\hat{h}_{k}(e)$. Then the length of each path $e_{k}$ is at most the length of $e$, which is less than $d_{\text {hyp }}\left(x^{*}, y^{*}\right)+2 \epsilon$. Taking a limit of the $e_{k}$, we get some curve $\hat{e}$, which joins $x^{*}$ and $y^{*}$, has length at most $d_{\text {hyp }}\left(x^{*}, y^{*}\right)+2 \epsilon$ and obviously lies in $W$.

This shows that any 2 points in $\Omega^{*}$ can be joined by a path in $W$, whose length is arbitrarily close to the distance between the points. Since $\Omega^{*}$ is open and $W$ is simply connected, this implies that $W$ contains the convex hull of $\Omega^{*}$; but because $\partial W \subset \partial \Omega^{*}$, we see that $W$ is the convex hull of $\Omega^{*}$. This completes the proof of the lemma.

Knowing now that $W$ is convex, in order to reach a contradiction assume that $W \neq U$. Then there is some hyperbolic line $L$, which contains a point in $\partial W$, say $p$, and has $W$ entirely on one side of it. (Through every point $p \in \partial W \cap U$ passes such a line $L$.) Let $L^{\prime}$ be the hyperbolic line that is an arc of a euclidean circle with euclidean radius 1 and whose euclidean midpoint $p^{\prime}$ lies on the negative real ray. Let $g$ be the hyperbolic orientation-preserving isometry that takes $L$ to $L^{\prime}$ and $p$ to $p^{\prime}$ and takes $W$ into the region to the left of $L^{\prime}$. (That is, $\operatorname{Re}(w)<\operatorname{Re}\left(p^{\prime}\right)$ for $w \in g(W)$.) Now let $q$ be the euclidean translation $q(z)=z+1-p^{\prime}$. Then $q$ takes $g(W)$ into $U$. Furthermore $q$ is clearly (strongly) expanding in the hyperbolic metric at points in $g(W)$ near $p^{\prime}$. Therefore the map $q \circ g$ takes $\Omega^{*}$ into $U$ and is expanding in the hyperbolic metric at some point of $\Omega^{*}$ near $p$, at $x^{*}=f(x)$, say. Let $\alpha>1$ be the expansion factor of $q \circ g$ at $x^{*}$. 
Since $f_{k} \rightarrow f$ and $f_{k}^{\prime} \rightarrow f^{\prime}$ as $k \rightarrow \infty$, uniformly on compact subsets of $\Omega$, the expansion factor of the map $f \circ f_{k}^{-1}$ at $f_{k}(x)$ tends to 1 . Let $k$ be large enough that this expansion factor $\beta$ is greater than $1 / \alpha$. Then the map $q \circ g \circ f \circ f_{k}^{-1}: f_{k}\left(\Omega_{k}\right) \rightarrow q\left(g\left(\Omega^{*}\right)\right)$ has an expansion factor $\beta \alpha>1$ at $f_{k}(x)$. But this contradicts the Schwarz-Pick lemma, Theorem 0.6, since the image of this map is contained in $U$, and $q$ and $g$ preserve circles. This contradiction completes the proof of the uniformization theorem in the hyperbolic case.

Parabolic Case $(f(\Omega)=\{0\})$. Define maps $g_{k}(z)=f_{k}(z) / f_{k}^{\prime}\left(z_{0}\right)$, $g_{k}: \Omega_{k} \rightarrow \mathbb{C}$. These maps clearly form a normal family, since $g_{k}^{\prime}\left(z_{0}\right)=1$. Therefore we assume without loss of generality that the $g_{k}$ converge uniformly on compact subsets of $\Omega$ to some map, say, $g$. Since $g^{\prime}\left(z_{0}\right)=1, g$ cannot be a constant and therefore is a conformal homeomorphism of $\Omega$ onto a domain, say, $\Omega^{*}$. As in the hyperbolic case, it follows from Corollary 6.2 that each boundary component of $\Omega^{*}$, with the possible exception of $g^{B}\left(K_{0}\right)$, is a circle or a point. We will show that $K_{0}^{*}=g^{B}\left(K_{0}\right)$ is a point (the point $\infty$ ), and then the proof will be complete. Striving for a contradiction, assume that $K_{0}^{*}$ consists of more than a single point and let $F$ be the connected component of $\hat{\mathbb{C}}-\Omega^{*}$ containing $K_{0}^{*}$.

We first consider the case where $F$ has interior points. Then there is a Möbius transformation $m$, which maps $\Omega^{*}$ into $U$, and with $\infty \in m(F)$. For each $k$ the map

$$
m \circ g \circ f_{k}^{-1}: f_{k}\left(\Omega_{k}\right) \rightarrow U
$$

satisfies the hypotheses of the Schwarz-Pick lemma, Theorem 0.6, and is therefore a contraction in the hyperbolic metric. This implies that the inverse map $f_{k} \circ g^{-1} \circ m^{-1}$ is an expansion, which clearly contradicts our assumption that $f_{k} \rightarrow 0$ as $k \rightarrow \infty$. The contradiction shows that int $F=\emptyset$.

The argument for the case where int $F=\emptyset$ is more involved, but will still use the Schwarz-Pick lemma. Let $D \subset \hat{\mathbb{C}}$ be some open geometric disk, whose closure $\bar{D}$ contains $F$ such that there are at least two distinct points, say, $p$ and $q$, in $\partial D \cap F$. One can take for $\bar{D}$, for example, the closed disk with minimal radius, which contains $F$, in the spherical metric. (We allow $\infty \in \bar{D}$.) We also assume that at least one of the two relatively open arcs of $\partial D$ with endpoints $p$ and $q$ is disjoint from $F$, as we may, without loss of generality. Let $\delta$ be such an arc.

For each angle $\theta$ let $\delta_{\theta}$ be the circular arc whose endpoints are $p$ and $q$, such that the oriented angle from $\delta$ to $\delta_{\theta}$ at $p$ is $\theta$. We take $\delta_{\theta}$ to be a relatively open arc; that is, $p, q \notin \delta_{\theta}$. For every $z \in \hat{\mathbb{C}}-\{p, q\}$ let $\theta(z)$ be that angle $\theta$ such that $z \in \delta_{\theta(z)}$. This defines $\theta(z)$ modulo $2 \pi$. However, since $\hat{\mathbb{C}}-F$ is simply connected, there is a continuous function $\hat{\theta}: \hat{\mathbb{C}}-F \rightarrow \mathbb{R}$ with $\hat{\theta}(z)=\theta(z)$ modulo $2 \pi$ for $z \in \hat{\mathbb{C}}-F$. 


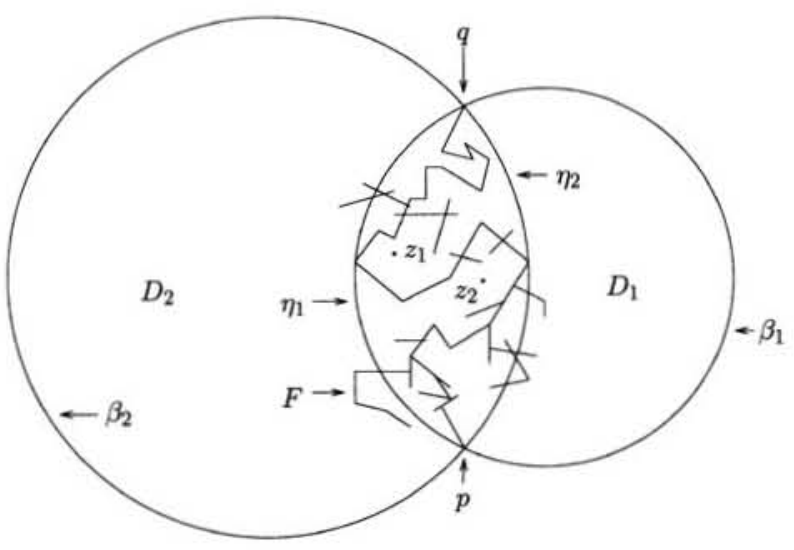

FIGURE 7.1

Now let $\theta_{1}=\inf \left\{\hat{\theta}(z): z \in \Omega^{*}\right\}=\inf \{\hat{\theta}(z): z \in \hat{\mathbb{C}}-F\}$, and let $\theta_{2}=$ $\sup \left\{\hat{\theta}(z): z \in \Omega^{*}\right\}=\sup \{\hat{\theta}(z): z \in \hat{\mathbb{C}}-F\}$. From the fact that $F \subset \bar{D}$ it follows that $\theta_{1}$ and $\theta_{2}$ are finite. Let $z_{1}$ be a point in $\Omega^{*}$ with $\hat{\theta}\left(z_{1}\right)<\theta_{1}+(\pi / 4)$ and let $z_{2} \in \Omega^{*}$ with $\hat{\theta}\left(z_{2}\right)>\theta_{2}-(\pi / 4)$. Let $\eta_{1}=\delta_{\theta_{1}}, \eta_{2}=\delta_{\theta_{2}}, \beta_{1}=\delta_{\theta_{1}+\pi}$ and $\beta_{2}=\delta_{\theta_{2}-\pi}$. Let $D_{1}$ and $D_{2}$ be the open disks

$$
D_{1}=\bigcup_{\theta_{1}<\theta<\theta_{1}+\pi} \delta_{\theta}, \quad D_{2}=\bigcup_{\theta_{2}-\pi<\theta<\theta_{2}} \delta_{\theta} .
$$

Then we have $z_{j} \in D_{j}, \partial D_{j}=\beta_{j} \cup \eta_{j} \cup\{p, q\}$, for $j=1,2$. Let $H_{1}$ be the connected component of $D_{1}-F$, which contains $z_{1}$, and let $H_{2}$ be the connected component of $D_{2}-F$, which contains $z_{2}$. (See Figure 7.1.)

Lemma 7.3. $\hat{\beta}_{1}=\partial H_{1}-F$ and $\hat{\beta}_{2}=\partial H_{2}-F$ are subarcs of $\beta_{1}$ and $\beta_{2}$, respectively.

Proof. Clearly $\partial H_{1}-F \subset \partial D_{1}$. From the definition of $\eta_{1}$ it follows that $\partial H_{1} \cap \eta_{1} \subset F$, and this gives $\partial H_{1}-F \subset \beta_{1}$.

Suppose now that $\partial D_{1} \neq \partial D$. Then the arc $\eta_{1}$ is contained in either $D$ or the complement of $\bar{D}$, because its endpoints $p$ and $q$ are in $\partial D$. But since there must be points $z \in F \subset \bar{D}$ with $\theta(z)$ arbitrarily close to $\theta_{1}$, and since $\eta_{1}=\delta_{\theta_{1}}$, it is impossible that $\eta_{1}$ is disjoint from $\bar{D}$. Therefore $\eta_{1} \subset D$ and $\beta_{1}$ is disjoint from $\bar{D}$, which gives $\beta_{1} \cap F=\emptyset$. This implies that $\hat{\beta}_{1}=\beta_{1}$ in the case where $\partial D \neq \partial D_{1}$.

If $\partial D_{1}=\partial D$, then we use the assumption that $\delta$ is an arc of $\partial D$ with endpoints $p$ and $q$, which is disjoint from $F$. Then $\delta$ must be equal to $\eta_{1}$ or $\beta_{1}$; and thus $F \cap \eta_{1}=\emptyset$ or $F \cap \beta_{1}=\emptyset$. If $F \cap \beta_{1}=\emptyset$, then we get again $\hat{\beta}_{1}=\beta_{1}$. Therefore suppose that $F \cap \eta_{1}=\emptyset$. (A priori this can happen.) From the definitions of $\theta_{1}$ and $\eta_{1}$ it then follows that $F$ must intersect $D_{1}$. 
Since $\partial D=\partial D_{1}$ and $F \subset \bar{D}$, this shows that $D=D_{1}$. This implies that $\hat{\beta}_{1}=\partial H_{1}-F$ is connected, because given 2 points $x, y \in \hat{\beta}_{1}$, one can connect them by a simple path $\gamma$ in $H_{1} \cup\{x, y\}$, and $F$, being connected, must be contained in one of the two connected components of $\overline{D_{1}}-\gamma$. This proves that $\hat{\beta}_{1}$ is a subarc of $\beta_{1}$; the proof for $\hat{\beta}_{2}$ is similar.

Returning to the proof of the Uniformization Theorem 7.1, we will now show that $H_{1} \cap H_{2}=\emptyset$. Observe that $\theta_{1}<\hat{\theta}(z)<\theta_{1}+\pi$ for $z \in H_{1}$, because $H_{1}$ is connected, $z_{1} \in H_{1} \subset D_{1}=\bigcup\left\{\delta_{\theta}: \theta_{1}<\theta<\theta_{1}+\pi\right\}$ and $\theta_{1}<\hat{\theta}\left(z_{1}\right)<$ $\theta_{1}+\pi / 2$. Similarly $\theta_{2}-\pi<\hat{\theta}(z)<\theta_{2}$ for $z \in H_{2}$. So it will be sufficient to show that $\theta_{1}+2 \pi \leqslant \theta_{2}$. For every $z \notin F$ we have $\theta_{1}<\hat{\theta}(z)<\theta_{2}$. Thus if $\theta$ is a number satisfying $\theta_{1}+2 \pi>\theta>\theta_{2}$, then $\theta$ cannot equal $\hat{\theta}(z)$ modulo $2 \pi$. Consequently $\bigcup\left\{\delta_{\theta}: \theta_{1}+2 \pi>\theta>\theta_{2}\right\} \subset F$. However, since $F$ has empty interior, this gives $\theta_{1}+2 \pi \leqslant \theta_{2}$, which establishes $H_{1} \cap H_{2}=\emptyset$.

Let $k$ be some integer such that $g^{-1}\left(z_{1}\right), g^{-1}\left(z_{2}\right) \in \Omega_{k}$. Let $A$ be a Jordan domain, which contains $g\left(\Omega_{k}\right)$ and whose boundary $\partial A$ is contained in $\Omega^{*}$. We also require that $\partial A$ intersect $\hat{\beta}_{1}$ in precisely 2 points $p_{1}, q_{1}$ and intersect $\hat{\beta}_{2}$ in precisely 2 points $p_{2}, q_{2}$. Thanks to Lemma 7.3 , it is not difficult to see that such a Jordan domain exists. (One can just take some Jordan curve in $\Omega^{*}$, which circles around $F$ very close to $F$, and then modify it, if necessary, to avoid access intersections with $\hat{\beta}_{1}$ and $\hat{\beta}_{2}$. The domain disjoint from $F$ bounded by this curve is taken as $A$.)

By the inductive hypothesis, there is a conformal map $f_{A}: A \cap \Omega^{*} \rightarrow U$, which takes each boundary component of $A \cap \Omega^{*}$ to a circle or a point, and with $f_{A}(\partial A)=\partial U$. Let $\alpha_{1}=\partial A \cap H_{1}, \alpha_{2}=\partial A \cap H_{2}, \beta=\hat{\beta}_{1} \cap A$ and $\eta=$ $\partial D_{1}-\beta$. Now we can apply the Angle Lemma 6.4 , with $f_{A}, A \cap \Omega^{*}, z_{1}, D_{1}, \alpha_{1}$ in place of $f, \Omega, z_{0}, D, \alpha$, respectively. We then conclude that

$$
\operatorname{ang}\left(f_{A}\left(z_{1}\right), f_{A}\left(\alpha_{1}\right)\right) \geqslant \operatorname{ang}\left(z_{1}, \eta\right)>\operatorname{ang}\left(z_{1}, \eta_{1}\right) .
$$

By similar reasoning we also have

$$
\operatorname{ang}\left(f_{A}\left(z_{2}\right), f_{A}\left(\alpha_{2}\right)\right)>\operatorname{ang}\left(z_{2}, \eta_{2}\right) .
$$

Because $\theta_{1}<\hat{\theta}\left(z_{1}\right)<\theta_{1}+(\pi / 4)$, it follows from Lemma 6.5 that $\operatorname{ang}\left(z_{1}, \eta_{1}\right)>$ $3 \pi / 2$. Likewise ang $\left(z_{2}, \eta_{2}\right)>3 \pi / 2$. From the above we conclude that

$$
\operatorname{ang}\left(f_{A}\left(z_{1}\right), f_{A}\left(\alpha_{1}\right)\right)>3 \pi / 2
$$

and

$$
\operatorname{ang}\left(f_{A}\left(z_{2}\right), f_{A}\left(\alpha_{2}\right)\right)>3 \pi / 2 \text {. }
$$


But $\alpha_{1} \cap \alpha_{2}=\emptyset$, because $H_{1} \cap H_{2}=\emptyset$; and therefore, $f_{A}\left(\alpha_{1}\right) \cap f_{A}\left(\alpha_{2}\right)=\emptyset$. It thus follows from (7.2) that

$$
\operatorname{ang}\left(f_{A}\left(z_{2}\right), f_{A}\left(\alpha_{1}\right)\right)<\pi / 2 .
$$

When compared with (7.1), this gives a lower bound $\mathrm{C}>0$ for the hyperbolic distance from $f_{A}\left(z_{1}\right)$ to $f_{A}\left(z_{2}\right)$ in the hyperbolic metric on $U$. The number $\mathrm{C}$ is an absolute constant, which can be described as the hyperbolic distance between two distinct circular $\operatorname{arcs}$ in $U$ that have common endpoints in $\partial U$, each having an angle of $\pi / 4$ with $\partial U$ (again by Lemma 6.5).

The domain $A \cap \Omega^{*}$ contains $g\left(\Omega_{k}\right)$, and so we can consider the map $f_{A}$ 。 $g \circ f_{k}^{-1}: f_{k}\left(\Omega_{k}\right) \rightarrow U$. By the Schwarz-Pick lemma, Theorem 0.6, it follows that this map is a contraction in the hyperbolic metric. This tells us that

$$
d_{\text {hyp }}\left(f_{k}\left(g^{-1}\left(z_{1}\right)\right), f_{k}\left(g^{-1}\left(z_{2}\right)\right)\right) \geqslant d_{\text {hyp }}\left(f_{A}\left(z_{1}\right), f_{A}\left(z_{2}\right)\right)>\mathrm{C},
$$

and $d_{\text {hyp }}\left(f_{k}\left(g^{-1}\left(z_{1}\right)\right), f_{k}\left(g^{-1}\left(z_{2}\right)\right)\right)>\mathrm{C}>0$ holds for every sufficiently large $k$. That is a contradiction to our assumption that $f_{k} \rightarrow 0$ as $k \rightarrow \infty$. And this contradiction completes the proof of the theorem.

\section{Domains in Riemann surfaces}

In this section we prove Theorem 0.2 . We will make use of the space of ends $\mathcal{E}(\Omega)$ of a Riemann surface $\Omega$, which is defined exactly as in Section 1. The term closed surface means a compact surface with no boundary and an open surface means a surface without boundary. We use the term "surface" to mean "connected surface".

Proof of Theorem 0.2. We start with the proof of existence. If the genus of $\Omega$ is 0 , then $\Omega$ can be conformally embedded in the Riemann sphere $\hat{\mathbb{C}}$, and existence follows from Theorem 0.1. Assume therefore that the genus is nonzero.

Because $\Omega$ has finite genus, there is some compact subset $F \subset \Omega$ such that each connected component of $\Omega-F$ is a 0 -genus (planar) surface, which has one boundary component in $\Omega$. It follows that there is a topological embedding $i: \Omega \rightarrow S$ of $\Omega$ into a closed topological surface such that every boundary component of $i(\Omega)$ in $S$ is contained in some topological disk in $S$. (One can "fill in the holes" in each connected component of $\Omega-F$.) Let $\tilde{S}$ be the universal cover of $S$, let $p: \tilde{S} \rightarrow S$ be the covering map and let $\tilde{\Omega}=p^{-1}(i(\Omega))$. Then $\tilde{\Omega}$ is a covering surface for $\Omega$, with covering map $p_{\Omega}=i^{-1} \circ p$, and thus has the structure of a Riemann surface. Moreover $\tilde{\Omega}$ is planar, since $\tilde{S}$ is a topological disk. From this it follows that $\tilde{\Omega}$ can be conformally embedded in the plane and, therefore, by Theorem $0.1, \tilde{\Omega}$ is conformally homeomorphic to a circle domain $\tilde{\Omega}^{*} \subset \mathbb{C}$. 
Let $\Gamma$ be the group of deck transformations for the covering $p: \tilde{S} \rightarrow S$. The group $\Gamma$ acts by conformal homeomorphisms on $\tilde{\Omega}$ and, via the conformal homeomorphism of $\tilde{\Omega}$ and $\tilde{\Omega}^{*}$, it also acts by conformal homeomorphisms on $\tilde{\Omega}^{*}$. Furthermore the uniqueness part in Theorem 0.1 shows that the action of $\Gamma$ on $\tilde{\Omega}^{*}$ is by Möbius transformations.

Suppose that $K$ is some boundary component of $\tilde{\Omega}$ in $\tilde{S}$. Since $p(K)$ is contained in a topological disk in $S$, it follows that no nontrivial element of $\Gamma$ stabilizes $K$. Let $e_{\infty}$ be the only end of $\tilde{S}$. Consequently no nontrivial element of $\Gamma$ fixes an end of $\tilde{\Omega}^{*}$, except for the end $e_{\infty}^{*}$, which corresponds to $e_{\infty}$. Let $E_{\infty}$ be the connected component of $\hat{\mathbb{C}}-\tilde{\Omega}^{*}$ corresponding to $e_{\infty}^{*}$ and let $\tilde{R}=\hat{\mathbb{C}}-E_{\infty}$. Then $\Gamma$ acts freely, co-compactly and discretely on $\tilde{R}$. The pair $\left(\tilde{R} / \Gamma, \tilde{\Omega}^{*} / \Gamma\right)$ is then the required pair.

To prove uniqueness let $R_{1}$ and $R_{2}$ be closed Riemann surfaces, let $\Omega_{1}, \Omega_{2}$ be circle domains in $R_{1}$ and $R_{2}$, respectively, and suppose that $h: \Omega_{1} \rightarrow \Omega_{2}$ is a conformal homeomorphism. Let $\tilde{R}_{1}, \tilde{R}_{2}$ be the universal covers of $R_{1}, R_{2}$, with covering maps $p_{1}, p_{2}$, respectively. We think of $\tilde{R}_{1} \subset \hat{\mathbb{C}}$ and $\tilde{R}_{2} \subset \hat{\mathbb{C}}$ as being the unit disk, the plane or the sphere. Set $\tilde{\Omega}_{1}=p_{1}^{-1} \Omega_{1}, \tilde{\Omega}_{2}=p_{2}^{-1} \Omega_{2}$; these are circle domains in $\hat{\mathbb{C}}$. From the fact that $\Omega_{1}$ and $\Omega_{2}$ are circle domains in $R_{1}$ and $R_{2}$, respectively, it follows that the homeomorphism $h$ lifts to a homeomorphism $\tilde{h}: \tilde{\Omega}_{1} \rightarrow \tilde{\Omega}_{2}$. From Theorem 0.1 we know that $\tilde{h}$ is a Möbius transformation. Therefore $\tilde{h}$ extends to a conformal homeomorphism $\tilde{H}$ : $\tilde{R}_{1} \rightarrow \tilde{R}_{2}$. From the fact that $\tilde{h}$ conjugates the deck transformations of the cover $p_{1}: \tilde{\Omega}_{1} \rightarrow \Omega_{1}$ to the deck transformations of the cover $p_{2}: \tilde{\Omega}_{2} \rightarrow \Omega_{2}$, it follows that $\tilde{H}$ conjugates the deck transformations of the cover $p_{1}: \tilde{R}_{1} \rightarrow R_{1}$ to the deck transformations of the cover $p_{2}: \tilde{R}_{2} \rightarrow R_{2}$ and therefore descends to a conformal homeomorphism $H: R_{1} \rightarrow R_{2}$. The restriction of $H$ to $\Omega_{1}$ is obviously $h$. This shows uniqueness, completing the proof of the theorem.

\section{Uniformizations of circle packings}

Surely circle packings and circle domains are closely related. The following definitions give a common generalization of these two concepts.

Definitions. Let $\Omega$ be any domain in $\hat{\mathbb{C}}$ (or, more generally, in a Riemann surface). A D-packing in $\Omega$ is an indexed collection $P=\left\{P_{i}: i \in V\right\}$ of compact topological disks in $\Omega$ with disjoint interiors. The nerve, or graph, of the packing $P$ is the abstract graph $G=(V, E)$, whose vertex set is $V$ and where an edge $\langle i, j\rangle$ occurs in $E$ precisely when the disks $P_{i}$ and $P_{j}$ intersect. An interstice of the packing is a connected component of $\Omega-\bigcup\left\{P_{v}: v \in V\right\}$, a finite interstice is an interstice whose boundary is contained in finitely many of the packed disks, and the carrier of the packing is the union of all the packed 
sets and all the finite interstices. A decent packing is a D-packing in which the intersection of any two sets is at most a single point, and the intersection of any three sets is empty. The limit points of a packing are the set of all points $p$ in $\widehat{\mathbb{C}}$ with the property that every neighborhood of $p$ intersects infinitely many of the packed sets. A packing $P$ in a domain $\Omega$ is said to be an acceptable packing in $\Omega$ if $P$ is decent and has no limit points in $\Omega$. If $P$ is an acceptable packing in $\Omega$, then $\Omega_{P}=\Omega-\bigcup\left\{\operatorname{int}\left(P_{i}\right): i \in V\right\}$ is a generalized domain; specifically it is the generalized domain associated with $P$ and $\Omega$.

Note that, in general, a generalized domain is not a domain, since it is not open. However it is connected and is the closure of its interior.

Given a D-packing $P$ in $\hat{\mathbb{C}}$, one can get a planar embedding of its graph. To do that choose a point in the interior of each packed set to be the image of the associated vertex. Then the image of each edge $\langle i, j\rangle$ can be chosen as a simple path that lies in $P_{i} \cup P_{j}$ and connects the images of the vertices. There is no problem in making the images of the edges disjoint, except at the vertices. Thus nerves of planar D-packings are planar.

Of particular interest are nerves that are maximal with respect to being planar; that is, the introduction of one additional edge to the graph would make it nonplanar. These are the 1-skeletons of a triangulation of an open planar surface. We will call them planar triangulations, for short, and when we use the term triangulation, it will be implicitly assumed that the triangulation is connected. Planar triangulations have another important property: their embedding in the sphere $\hat{\mathbb{C}}$ is topologically unique (or unique up to reflection, if the orientation of the sphere is taken into account). This means that any two embeddings of a planar triangulation in $\hat{\mathbb{C}}$ are related by a self-homeomorphism of $\hat{\mathbb{C}}$. Thus a decent packing in $\hat{\mathbb{C}}$, whose nerve is a triangulation, is topologically determined by its nerve. We will see below that when the packed sets are geometric disks and the nerve is a given planar triangulation, then under certain conditions the packing is also geometrically determined.

One can study either a packing or the generalized domain associated to it. The difference is like the proverbial difference between looking at the halffull glass or at the half-empty glass. Of course, when there are few edges in the nerve of the packing, there is little to work with, and one must look at the domain. Historically both approaches to the subject are present. For example, Koebe looked at the domain and achieved the circle packing theorem as a consequence of his uniformization theorem. On the other hand, Thurston mostly looked at the circles, while the Rodin-Sullivan work [RoSu] and the paper [He2] adopt a mixture of the two views. In retrospect, the incompatibility theorem, which is the main tool in [Sch2] and [Sch3], can be seen as some kind of fixed-point theorem for packings. There, two finite topological 
packings satisfy some boundary conditions analogous to the condition that the associated map on the boundary of the domain have a fixed-point index -1 . The conclusion is that there exists a "fixed point of negative index" between the packings.

Definitions. A conformal homeomorphism between generalized domains $h: \Omega \rightarrow \Omega^{*}$ is a homeomorphism that is conformal in the interior of $\Omega$, while an anticonformal homeomorphism is a homeomorphism that is anticonformal in the interior of $\Omega$. If such an $h$ exists, then $\Omega$ and $\Omega^{*}$ are said to be conformally or anticonformally homeomorphic, respectively.

Example 9.1. Let $P$ and $P^{*}$ be decent packings in $\hat{\mathbb{C}}$ having carriers $\Omega$ and $\Omega^{*}$ and planar triangulations $\mathcal{T}$ and $\mathcal{T}^{*}$ as nerves, respectively. Then $P$ and $P^{*}$ are acceptable packings in $\Omega$ and $\Omega^{*}$, respectively; and the associated generalized domains $\Omega_{P}$ and $\Omega_{P}^{*}$. are conformally homeomorphic or anticonformally homeomorphic if and only if $\mathcal{T}$ and $\mathcal{T}^{*}$ are combinatorially isomorphic. To see this, note that all of the interstices in the packings must be triangular interstices; that is, their boundary lies on three of the packed sets. Thus all one has to do to show that $\Omega_{P}$ and $\Omega_{P *}^{*}$ are conformally or anticonformally homeomorphic is to construct the conformal, or anticonformal, maps between combinatorically corresponding triangular interstices and glue them properly. Since there is a freedom of choice of the image of three points on the boundary for Riemann maps between Jordan domains, one can do this while maintaining the continuity at the points of contact between any two interstices. This shows that $\Omega$ and $\Omega^{*}$ are conformally or anticonformally homeomorphic. The other direction is obvious.

When we speak of a circle packing, we will mean a D-packing of geometric, rather than topological, closed disks. If a circle packing has no limit points in a domain $\Omega$, then it follows that it is acceptable in $\Omega$. A circle packing, whose nerve is a triangulation, is always acceptable in its carrier.

Definition. Let $\Omega$ be a circle domain and let $P$ be an acceptable circle packing in $\Omega$. The associated generalized domain $\Omega_{P}$ will be called a generalized circle domain.

We can now state a generalization of Theorem 0.1 , which is applicable to circle packings.

Theorem 9.2. Any generalized domain $\Omega$ in $\hat{\mathbb{C}}$ that has at most countably many ends is conformally homeomorphic to a generalized circle domain $\Omega^{*} \subset$ $\hat{\mathbb{C}}$. Moreover $\Omega^{*}$ is unique up to Möbius transformations, and every conformal automorphism of $\Omega^{*}$ is the restriction of a Möbius transformation. 
Theorem 0.3 follows immediately as a corollary:

Proof of Theorem 0.3 . We start with existence. One easily constructs a decent planar packing $P^{*}$ with nerve $\mathcal{T}$. Since $P^{*}$ is acceptable in its carrier $\Omega^{*}$, one can form the generalized domain $\Omega_{P^{*}}^{*}$. From Theorem 9.2 we conclude that there is a generalized circle domain $\Omega \subset \hat{\mathbb{C}}$ that is conformally homeomorphic to $\Omega_{P}^{*}$. The circle packing associated to $\Omega$ is then the required packing $P$. This proves existence. The uniqueness follows from Example 9.1 and Theorem 9.2 .

To prove Theorem 9.2 one must essentially adapt, for generalized domains, the proof of Theorem 0.1 and the proofs of all the theorems that precede it. There are two minor difficulties, which require some changes in the proofs. The first has to do with the fact that the interior of a generalized domain is not connected. When working with domains, we used the fact that if a conformal function has nonisolated fixed points, then it is the identity. This is no longer true when the domain of the function is not connected and, therefore, in the proof of Theorem 9.2, one must take special care to avoid nonisolated fixed points.

In the proofs above, quite often we have chosen a Jordan curve $\gamma$ in the domain $\Omega$ to cut and isolate a part of the domain we wanted to examine from other parts. This can still be done in generalized domains, but the resulting two pieces that $\Omega$ breaks into may no longer be generalized domains. An example of this phenomenon can be seen in the generalized domain $\Omega_{H}$ obtained from the plane by the deletion of the interiors of disks that form an infinite hexagonal circle packing. The bounded part of $\Omega_{H}$ determined by any Jordan curve $\gamma \subset \Omega_{H}$ will not be a generalized domain, unless it is contained in one interstice, because $\gamma$ would have to touch some boundary circles more than once. This forces us to further broaden the class of "domains" under discussion.

Definitions. Let $\hat{\Omega}$ be some domain in $\hat{\mathbb{C}}$ and let $P$ be a D-packing in $\hat{\Omega}$. Suppose that the intersection of any three sets in the packing $P$ is empty and the intersection of any two contains at most a finite number of points. Further suppose that at most one of the connected components of the complement of any pair of sets in $P$ intersects with other sets in the packing. Then $\Omega=$ $\hat{\Omega}-\bigcup\left\{\operatorname{int}\left(P_{v}\right): v \in V\right\}$ will be called a degenerated generalized domain.

A bi-gon in a degenerated generalized domain is a finite interstice whose boundary lies in two of the sets in the packing. Thus a degenerate generalized domain without bi-gons is a generalized domain.

A morphism of degenerate generalized domains is a continuous map $f: \Omega \rightarrow \Omega^{*}$ between degenerate generalized domains that is conformal and 
injective in $\operatorname{int}(\Omega)-B$, where $B$ is some union of bi-gons of $\Omega$ and $f$ is constant in each bi-gon contained in $B$.

As explained above, the advantage of working with degenerated generalized domains over generalized domains is that it is easy to cut a degenerated generalized domain along a Jordan curve and get two degenerated generalized domains.

Proof of Theorem 9.2. Let $\hat{\Omega}$ and $P=\left(P_{i}: i \in V\right)$ be the domain and the packing, which define $\Omega$; that is, $\Omega=\hat{\Omega}-\bigcup\left\{\operatorname{int}\left(P_{i}\right): i \in V\right\}$.

We need a construct for degenerated generalized domains analogous to the space of boundary components of domains. The elements of this space will be called the boundary elements. These come in two flavors: the elements at infinity are just the boundary components of $\hat{\Omega}$, and the border elements are the sets of the form $\partial P_{i}$. The topology on the set of boundary elements $B^{e}(\Omega)$ is such that any neighborhood in $B^{e}(\Omega)$ around any element at infinity $K \in B(\hat{\Omega})$ is the set of all boundary elements that intersect a neighborhood of $K$ in $\hat{\mathbb{C}}$, and $\left\{\partial P_{i}\right\}$ is a neighborhood of any border element $\partial P_{i}$. (Thus the set of border elements is discrete in $B^{e}(\Omega)$, and the inclusion of $B(\hat{\Omega})$ in $B^{e}(\Omega)$ is a homeomorphic embedding.) As with $B(\hat{\Omega})$, the set $B^{e}(\Omega)$ is compact, Hausdorff and countable. The type and rank are then defined for $B^{e}(\Omega)$ as for $B(\hat{\Omega})$.

In the following paragraphs we outline the modifications needed in the theorems and lemmas leading to Theorem 0.1 to get corresponding statements for degenerate generalized domains.

Note that Theorem 2.1 also holds if we allow the boundary of $A$ to be a finite union of Jordan curves, which may intersect at finitely many points.

We will need a slightly more general version of Lemma 2.2 . In the more general version we do not require $f$ to be a homeomorphism, but we do require the preimage of any point in $K$ to be a point or an arc on $J$. The hypothesis that $f$ is orientation preserving should then be weakened to the requirement that the image of a positively oriented arc from a point $x$ to a point $y$ in $J$ be a positively oriented arc from $f(x)$ to $f(y)$ in $J$, or a single point. In this situation part (1) of Lemma 2.2 is dropped, the proof for the case $J \subset \hat{K}$ in part (2) remains unchanged and the proof for the case $K \subset \hat{J}$ is done similarly as the proof for $J \subset \hat{K}$. The proofs of parts (3) and (4) remain unchanged.

Some adjustments are needed in Corollary 2.4 as well. First $\Omega$ and $\Omega^{*}$ are permitted to be possibly degenerated generalized domains, with $f$ a morphism between them. Obviously any mention of boundary components is replaced by boundary elements. (This same change is needed in all of the lemmas and theorems we discuss here and will not be mentioned, unless there is some 
special need.) Of course we no longer require that $F$, the continuous extension of $f$, be a homeomorphism, since $f$ does not have to be a homeomorphism, but only that the restriction of $F$ to each boundary element at infinity be a homeomorphism. The conclusions of the corollary also need some revision. The new conclusions are that $f$ has at most $n$ isolated fixed points and the number of fixed points in any set $S$ of isolated fixed points is at most $n$, counting multiplicities. This change is needed, since $f$ may fix whole connected components of the interior of $\Omega$. The proof remains almost unchanged. One only needs to note that $z \rightarrow F(z)+\mathrm{c}$ has only isolated fixed points if it has no fixed points on the boundary.

The description of $\Omega$ in the statement of the Schwarz-Pick lemma, Theorem 0.6 , is modified to the following: $\Omega$ is a possibly degenerate generalized domain contained in $\bar{A}$, and every boundary element of it is a circle or a point, except possibly for $\partial A$, which is also a boundary element of $\Omega$. A similar change is done for $\Omega^{*}$. The statement of Lemma 4.1 changes only in that the continuous extension of $f$ to $\bar{\Omega}$ is not required to be a homeomorphism, only its restriction to each boundary element at infinity needs to be a homeomorphism. A change is needed in the proof of this lemma, since when one of the fixed points $p, q$ is a nonisolated fixed point, one cannot immediately conclude that $f$ is the identity. Suppose this to be the case. Then $f$ must fix a connected component of the interior of $\Omega$ that is not a bi-gon. Let $H$ be the union of the connected components that $f$ fixes. If $H$ is the interior of $\Omega$, the lemma follows; if not, then there is some border boundary element $K$ that has nontrivial arcs in $\bar{H}$ and in the closure of some connected component $B$ of the interior of $\Omega$ which is not in $H$. Since $f$ fixes an $\operatorname{arc}$ of $K$, then $f(K)=K$, because both are circles. If $f$ is the identity on $K$, then $f$ must fix $B$, which contradicts our assumptions. If not, then there will be two points, $x, y \in K$, such that $d_{\text {hyp }}(x, y)<d_{\text {hyp }}(f(x), f(y))$. The same would hold for some points $x^{\prime}, y^{\prime}$ in the interior of $\Omega$ sufficiently close to $x, y$, respectively. Then one can postcompose $f$ with a Möbius transformation $m$, which contracts distances in the hyperbolic metric on $U$ and takes $f\left(x^{\prime}\right)$ and $f\left(y^{\prime}\right)$ to $x^{\prime}$ and $y^{\prime}$, respectively. Since one has 2 dimensions of freedom in choosing $m$, one easily arranges that $x^{\prime}$ and $y^{\prime}$ will be isolated fixed points of $m \circ f$. Then the contradiction follows as in the original proof of Lemma 4.1.

The statement and proof of Lemma 5.1 for possibly degenerated generalized domains remain essentially unchanged.

In the formulation of Lemma 5.2, again the requirement that the continuous extension of $f$ to $\bar{\Omega}-K$ be a homeomorphism needs to be changed to the requirement that its restriction to each boundary element at infinity $H \in B_{\infty}^{e}(\Omega)$ be a homeomorphism. In the proof, the only modification is that 
one must make sure that $p$ is an isolated fixed point for $g$. It is easy to see that $p$ can be chosen so that this is the case. (Note that $p$ is chosen before $g$, and a different choice of $p$ may give a different choice of $g$.)

The statement and proof of the Boundary Extension Theorem remain essentially the same, and the generalized form of Theorem 0.6 follows from it and Lemma 4.1. The uniqueness part of Theorem 9.2 now clearly follows from Theorem 0.6.

Except for the obvious modifications, similar to those in the Schwarz-Pick lemma (Theorem 0.6), no change is needed in the formulation of the Maximum Modulus Theorem 6.1. In the proof, one must take care of the possibility that $z_{0}$ is a nonisolated fixed point of $g$. In that case, let $H$ be the union of the connected components of the interior of $\Omega$ that are fixed by $g$. Obviously $H$ cannot contain all of the interior of $\Omega$ and, therefore, there is a connected component $B$ of the interior of $\Omega$ that is disjoint from $H$, but whose closure intersects the closure of $H$. If $p$ is in the intersection of the closures, then in $B$ near $p$ one can find a point $q$ with $g(q)-q$ very close to 0 . Furthermore $q$ can be chosen to also satisfy $g^{\prime}(q) \neq 1$. Then $q$ is an isolated fixed point for $\hat{g}(z)=g(z)-g(q)+q$, and a contradiction follows.

Corollary 6.2 requires substantial changes, and it will be replaced by a discussion below.

The changes necessary in the formulation and proof of the Angle Lemma 6.4 are similar to those made in the previous lemmas and theorems above. These changes are left to the reader.

In the modified proof of existence, the inductive claim is that given a degenerate generalized domain $\Omega \subset \hat{\mathbb{C}}$ with $\operatorname{tp}(\Omega)<(\lambda, n)$ there exists a morphism of it onto a generalized circle domain. It is not very well known, but Koebe [Ko4] also proved the existence statement in the case of degenerate generalized domains with finitely many boundary elements by taking limits of complements of packings, where the packed disks almost touch. This covers the base of the induction.

The maps $f_{k}$ are defined as in the original version, but one has to work a little harder to argue that their limits are either a constant or a morphism. First it is clear that a subsequence of the $f_{k}$ converges uniformly on compact subsets of the interior of $\Omega$. Using the reflection principle, one easily concludes that a subsequence of $\left\{f_{k}\right\}$ converges uniformly on compact subsets of $\Omega$. Then exactly the same argument as in Corollary 6.2 shows that $f^{B}(K)$ is a circle or a point whenever $K \in B^{e}(\Omega)-K_{0}$, where $f$ is the limit of the $f_{k}$.

We will now show that $f$ is either a constant or a morphism. Restricted to each connected component of the interior of $\Omega, f$ is either a constant or a conformal homeomorphism. Also $f$ is clearly injective where it is not a constant. Suppose that $f$ is constant on some interstice $L$, which is not a 
bi-gon. Say it takes the value c there. Let $M$ be the connected component of $f^{-1}(\mathrm{c})$ containing $L$ and let $\partial_{\Omega} M$ be the relative boundary of $M$ in $\Omega$. If $K$ is some border boundary element contained in $M$, then clearly every connected component of the interior of $\Omega$ whose boundary has an arc on $K$, is also contained in $M$. This shows that $\partial_{\Omega} M$ consists of contact points; that is, points in the intersection of the closures of two distinct interstices. The number of points in $\partial_{\Omega} M$ cannot be 1 ; on the other hand, if there are 2 or more contact points in $\partial_{\Omega} M$, then there are at least 3 border boundary elements intersecting $\partial_{\Omega} M$. These elements will have the property that their images under $f$ contain c, but also contain other points. However this is impossible, since at most two circles can touch at any given point. This implies that $\partial_{\Omega} M$ is empty and thus shows that $f$ is a constant if it is constant on some connected component of the interior of $\Omega$ that is not a bi-gon.

This same argument is repeated for the hyperbolic and parabolic cases. Except for this, the proof remains intact. This completes the proof of Theorem 9.2.

Proof of Theorem 0.4. Theorem 0.4 follows from Theorem 9.2 exactly as Theorem 0.2 followed from Theorem 0.1 .

\section{Addendum: Almost circular domains with uncountably many boundary components}

We now state and outline the proof of a generalization to the existence part of our main result, Theorem 0.1, which was obtained after this article was accepted. The details will appear in a forthcoming paper.

Theorem 10.1. Let $\Omega$ be a domain in $\mathbb{C}$ and let $B_{*}(\Omega) \subset B(\Omega)$ be the collection of all boundary components that are not circles or points. If the closure of $B_{*}(\Omega)$ in $B(\Omega)$ is countable, then $\Omega$ is conformally homeomorphic to a circle domain.

The proof of Theorem 10.1 proceeds by induction in much the same way as the proof of existence presented earlier. The sticky point is, however, that the rigidity results used, primarily the Schwarz-Pick lemma, require some extra hypotheses when there are uncountably many boundary components. For this purpose, the theory of quasiconformal maps is useful. More specifically the Schwarz-Pick lemma, and most of the other results here, are applications of Corollary 2.4, which in general fails when there are uncountably many boundary components. The first step in the proof of Theorem 10.1 is a variation of that corollary, as follows: 
LEMMA 10.2. Let $f: \Omega \rightarrow \Omega^{*}$ be a conformal homeomorphism between bounded plane domains and let $B_{0} \subset B(\Omega)$ be a finite collection of boundary components of $\Omega$. Suppose that $f$ extends to a quasiconformal homeomorphism $F: \mathbb{C} \rightarrow \mathbb{C}$ and that the following conditions hold:

(1) the conformal dilatation of $F$ on $\partial \Omega$ is 0 a.e. (this is automatically satisfied if $\partial \Omega$ has measure 0$)$;

(2) all of the boundary components in $B(\Omega)-B_{0}$ and $B\left(\Omega^{*}\right)-f^{B}\left(B_{0}\right)$ are circles and points, and all of the boundary components in $B_{0}$ are Jordan curves;

(3) $B_{0}$ contains the boundary component of $\Omega$, which is contained in the unbounded component of $\mathbb{C}-\Omega$ and, similarly, $f^{B}\left(B_{0}\right)$ contains the boundary component of $\Omega^{*}$, which is contained in the unbounded component of $\mathbb{C}-\Omega^{*}$;

(4) $F$ has no fixed points in any of the boundary components in $B_{0}$.

Let $n$ be the index of the restriction of $F$ to the boundary components in $B_{0}$. Then $f$ has at most $n$ fixed points in $\Omega$. Furthermore, if $S$ is a set of fixed points of $f$, then the total number of fixed points for $f$ in $S$, counting multiplicity, is at most $n$.

The proof of this lemma is based on approximations by finitely connected domains, on Corollary 2.4 for finitely connected domains and on a rigidity result of Sullivan $[\mathrm{Su}]$ to show that the approximations converge to $f$.

The rest of the proof of Theorem 10.1 is like the proof of the existence part of Theorem 0.1, but with the extra burden that whenever Corollary 2.4 is directly or indirectly applied, it can be arranged that the hypotheses of Lemma 10.2 are satisfied.

Princeton University, Princeton, New Jersey

Weizmann Institute, Rehovoth, IsRael

\section{REFERENCES}

[A] D. Aharonov, The hexagonal packing lemma and discrete potential theory, Can. Math. Bull. 33 (1990), 247-252.

[AhBe] L. Ahlfors and L. Bers, Riemann's mapping theorem for variable metrics, Ann. of Math. 72 (1960), 385-404.

[An1] E.M. ANDREev, On convex polyhedra in Lobačevskiľ spaces, Mat. Sb. (N.S.) 81 (1970), 445-478; English transl. in Math. USSR Sb. 10 (1970), 413-440.

[An2] — On convex polyhedra of finite volume in Lobačevskiǔ space, Mat. Sb. (N.S.) 83 (1970), 256-260; English transl. in Math. USSR Sb. 12 (1970), 255-259.

[BaFP] I. BÁRÁNY, Z. FÜrEDI and J. PACH, Discrete convex functions and proof of the six circle conjecture of Fejes Tóth, Can. J. Math. 36 (1984), 569-576. 
[BSte1] A.F. Beardon and K. Stephenson, The uniformization theorem for circle packings, Indiana Math. J. 39 (1990), 1383-1425.

[BSte2] — Circle packings in different geometries, Tôhoku Math. J. 43 (1991), 27-36.

[BSte3] The Schwarz-Pick lemma for circle packings, Illinois J. of Math. 35 (1991), $577-606$.

[Be] L. Bers, Uniformization by Beltrami equations, Comm. Pure Appl. Math. 14 (1961), 215-228.

[BoS] P.L. Bowers and K. Stephenson, The set of circle packing points in the Teichmüller space of a surface of finite conformal type is dense, Math. Proc. Cambridge Phil. Soc. 111 (1992), 487-513.

[Br1] R. BRooKs, On the deformation theory of classical Schottky groups, Duke Math. J. 52 (1985), 1009-1024.

[Br2] Circle packings and co-compact extensions of Kleinian groups, Invent. Math. 86 (1986), 461-469.

[CR] I. CARTER and B. RODIN, An inverse problem for circle packing and conformal mapping, Preprint.

[CdV1] Y. COLIN DE Verdière, Empilements de cercles: Convergence d'une méthode de point fixe, Forum Math. 1 (1989), 395-402.

[CdV2] Un principe variationnel pour les empilements de cercles, Invent. Math. 104 (1991), 655-669.

[De] R. Denneberg, Konforme Abbildung einer Klasse unendlich vielfach zusammenhangender schlichter Bereiche auf Kreisbereiche, Diss., Leipziger Berichte 84 (1932), 331-352.

[Gr] H. GröтzsCH, Eine Bemerkung zum Koebeschen Kreisnormierungsprinzip, Leipziger Berichte 87 (1935), 319-324.

[GPo] V. Guillemin and A. Pollack, Differential Topology, Prentice-Hall, Englewood Cliffs, 1974, p. 222.

[Haa] A. HAAS, Linearization and mappings onto pseudocircle domains, Trans. A.M.S. 282 (1984), 415-429.

[Hau] F. Hausdorff, Set Theory, $2^{\text {nd }}$ ed., Chelsea Pub. Co., New York, 1962.

[He1] Z.X. HE, Solving Beltrami equations by circle packings, Trans. A.M.S. 322 (1990), $657-670$.

[He2] _ An estimate for hexagonal circle packings, J. Diff. Geom. 33 (1991), 395-412.

[HeSch] Z.X. He and O. Schramm, A hyperbolicity criterion for circle packings, Preprint.

[Ko1] P. KoeвE, Uber die Uniformisierung beliebiger analytischer Kurven, III, Nachr. Ges Wiss. Gott. (1908), 337-358.

[Ko2] — Abhandlungen zur Theorie der Konformen Abbildung: VI. Abbildung mehrfach zusammenhängender Bereiche auf Kreisbereiche, etc., Math. Z. 7 (1920), 235-301.

[Ko3] — Uber die konforme Abbildung endlich- und unendlich-vielfach zusammenhängender symmetrischer Bereiche auf Kreisbereiche, Acta Math. 43 (1922), 263-287.

[Ko4] Kontaktprobleme der konformen Abbildung, Berichte Verhande. Sächs. Akad. Wiss. Leipzig, Math.-Phys. Klasse 88 (1936), 141-164.

[LV] O. Lehto and K.I. Virtanen, Quasiconformal Mappings in the Plane, Springer-Verlag, Berlin, 1973, p. 258.

[MRo] A. MARDEn and B. Rodin, On Thurston's formulation and proof of Andreev's theorem, in Computational Methods and Function Theory, Ruscheweyh, Saff, Salinas, Varga, eds., Lecture Notes in Math. 1435, Springer-Verlag, 1989, pp. 103-115.

[Me1] H. Meschowski, Uber die konforme Abbildung gewisser Bereiche von unendlich hohen Zusammenhang auf Vollkreisbereiche, I, Math. Ann. 123 (1951), 392-405.

[Me2] U Uber die konforme Abbildung gewisser Bereiche von unendlich hohen Zusammenhang auf Vollkreisbereiche, II, Math. Ann. 124 (1952), 178-181.

[Mi] J.W. Milnor, Topology from the Differential Viewpoint, Univ. Press of Virginia, 1965, p. 64 . 
[Ro1] B. Rodin, Schwarz's lemma for circle packings, Invent. Math. 89 (1987), 271-289.

[Ro2] — Schwarz's lemma for circle packings, II, J. Diff. Geom. 30 (1989), 539-554.

[Ro3] - On a problem of A. Beardon and K. Stephenson, Indiana Math. J. 40 (1991), 271-275.

[RoSu] B. Rodin and D. Sullivan, The convergence of circle packings to the Riemann mapping, J. Diff. Geom. 26 (1987), 349-360.

[Sa] L. Sario, Uber Riemannsche Flachen mit hebbarem Rand, Ann. Acad. Sci. Fenn., Ser. AI 50 (1948), 1-79.

[Sch1] O. Schramm, Packing Two-dimensional Bodies with Prescribed Combinatorics and Applications to the Construction of Conformal and Quasiconformal Mappings, Ph.D. Thesis, Princeton Univ., 1990.

[Sch2] — Rigidity of infinite (circle) packings, J. A.M.S. 4 (1991), 127-149.

[Sch3] _ Existence and uniqueness of packings with specified combinatorics, Israel J. Math. 73 (1991), 321-341.

[Si1] R.J. SiBneR, Uniformizations of symmetric Riemann surfaces by Schottky groups, Trans. A.M.S. 116 (1965), 79-85.

[Si2] , Remarks on the Koebe Kreisnormierungsproblem, Comm. Math. Helv. 43 (1968), 289-295.

[Si3] 'Uniformizations' of infinitely connected domains, in Advances in the Theory of Riemann Surfaces, Proc. 1969 Stony Brook Conf., Ann. of Math. Studies 66, Princeton Univ. Press, 1971, pp. 407-419.

[Ste] K. Stephenson, Circle packings in the approximation of conformal mappings, Bull. A.M.S. 23 (1990), 407-415.

[Str1] K.L. Strebel, Uber das Kreisnormierungsproblem der konformen Abbildung, Ann. Acad. Sci. Fenn. 1 (1951), 1-22.

[Str2] — Uber die konforme Abbildung von Gebieten unendlich hohen Zusammenhangs, Comm. Math. Helv. 27 (1953), 101-127.

[Su] D. Sullivan, On the ergodic theory at infinity of an arbitrary discrete group of hyperbolic motions, Ann. of Math. Studies 97, Princeton Univ. Press, 1981, pp. $465-496$.

[Th1] W.P. Thurston, The geometry and topology of 3-manifolds, Lecture notes, Princeton Univ., 1980, Chapter 13.

[Th2] — The finite Riemann mapping theorem, invited address, International Symposium in Celebration of the Proof of the Bieberbach Conjecture, Purdue University, 1985 .

(Received July 8, 1991) 